\title{
Role of nanoparticles in osteogenic differentiation of bone marrow mesenchymal stem cells
}

\author{
Nadia S. Mahmoud (1) - Hanaa H. Ahmed (1) - Mohamed R. Mohamed (D) \\ Khalda S. Amr (D) Hadeer A. Aglan (iD) Mohamed A. M. Ali - Mohamed A. Tantawy $(\mathbb{D}$
}

Received: 1 August 2019/Accepted: 2 November 2019/Published online: 13 November 2019

(C) Springer Nature B.V. 2019

\begin{abstract}
The present study aimed to investigate the osteoinductive potentiality of some selected nanostructures; Hydroxyapatite (HA-NPs), Gold (Au-NPs), Chitosan (C-NPs), Gold/hydroxyapatite (Au/HA-NPs) and Chitosan/hydroxyapatite (CH-NPs) on bone marrow- derived mesenchymal stem cells (BM-MSCs). These nanostructures were characterized using transmission electron microscope and Zetasizer. MSCs were isolated from bone marrow of rat femur bones and their identity was documented by morphology, flow cytometry and multi-potency capacity. The influence of the selected nanostructures on the viability, osteogenic differentiation and subsequent matrix mineralization of BM-MSCs was determined by MTT
\end{abstract}

N. S. Mahmoud $(\bowtie) \cdot$ H. H. Ahmed ·

H. A. Aglan · M. A. Tantawy

Hormones Department, Medical Research Division,

National Research Centre, 33 EL Bohouth St. (former EL

-Tahrir st.), Dokki, Giza P.O. 12622, Egypt

e-mail: nadiasamy@sci.asu.edu.eg

H. H. Ahmed

e-mail: hh.ahmed@nrc.sci.eg

H. A. Aglan

e-mail: ha.ajlan@nrc.sci.eg

M. A. Tantawy

e-mail: mohamed_tantawy@daad-alumni.de

N. S. Mahmoud · H. H. Ahmed · H. A. Aglan ·

M. A. Tantawy

Stem Cells Lab, Center of Excellence for Advanced

Sciences, National Research Centre, Dokki, Giza, Egypt assay, molecular genetic analysis and alizarin red $\mathrm{S}$ staining, respectively. MTT analysis revealed insignificant toxicity of the tested nanostructures on BMMSCs at concentrations ranged from 2 to $25 \mu \mathrm{g} / \mathrm{ml}$ over $48 \mathrm{~h}$ and $72 \mathrm{~h}$ incubation period. Notably, the tested nanostructures potentiate the osteogenic differentiation of BM-MSCs as evidenced by a prominent over-expression of runt-related transcription factor 2 (Runx-2) and bone morphogenetic protein 2 (BMP-2) genes after 7 days incubation. Moreover, the tested nanostructures induced matrix mineralization of BMMSCs after 21 days as manifested by the formation of calcium nodules stained with alizarin red S. Conclusively, these data provide a compelling evidence for

M. R. Mohamed · M. A. M. Ali

Biochemistry Department, Faculty of Science, Ain Shams

University, Cairo, Egypt

e-mail: mohamed_elsotohi@sci.asu.edu.eg

M. A. M. Ali

e-mail: mohd_ali2@sci.asu.edu.eg

K. S. Amr

Medical Molecular Genetics Department, Human

Genetics and Genome Researches Division, National

Research Centre, Dokki, Giza, Egypt

e-mail: khalda_nrc@yahoo.com 
the functionality of the studied nanostructures as osteoinductive materials motivating the differentiation of BM-MSCs into osteoblasts with the most prominent effect observed with Au-NPs and Au/HANPs, followed by CH-NPs.

Keywords Bone marrow mesenchymal stem cells . Osteogenic differentiation · Hydroxyapatite $\cdot$ Gold · Chitosan $\cdot$ Nanoparticles

\section{Introduction}

During the last decades, mesenchymal stem cells afforded a great cell source for regenerative medicine and tissue engineering (Linard et al. 2018). In recent years, bone marrow-derived mesenchymal stem cells (BM-MSCs) have drawn much interest in most researches owing to their unique characteristics such as proliferative, self- renewal ability and low immunogenicity (Wang et al. 2016). BM-MSCs are capable of differentiation into three different mesodermal lineages (adipogenic, chondrogenic and osteogenic) upon exposure to specific inducing factors in their microenvironment. Such driving differentiation ability towards desired lineages have been extensively employed in the context of tissue engineering whereby differentiated cells are implanted at the site of injury favoring tissue repair or replacement (Ullah et al. 2015). BM-MSCs express a cluster of cell surface antigens showing positive expression of CD73, CD90 and CD105 and negative expression of CD34, CD45, CD14 and CD19 (Müller et al. 2018). Upon the intravenous injection of BM-MSCs into experimental animals suffering from tissue injury, they will migrate to the site of injury where they trigger tissue repair via enhancing the expression of various growth factors (Fitzsimmons et al. 2018).

Therapeutic modalities that target bone formation process, either by increasing the resident osteoblasts' population or stimulating their activity, could offer an interesting approach for promoting both bone formation and regeneration. Bone regeneration through MSCs induction could induce osteogenesis and provide a therapeutic strategy for mitigating agerelated osteoporosis (Yao et al. 2013). Both autologous and allogeneic transplantation of stem cells have been extensively used for the treatment of cardiovascular diseases (Müller et al. 2018) and neurodegenerative diseases (Mahla 2016). However, in vivo transplantation of MSCs was unable to motivate osteogenesis in bone owing to the nonspecificity of MSCs and their failure to be recruited to the bone surface unless it was subjected to injury (Yao and Lane 2015).

Nanoscale biomaterials have made a great progress in many biomedical applications due to their unique characteristics, including outstanding mechanical strength and biodegradability, compared to those of micro- and macro-structures (Roohani-Esfahani et al. 2010; Mauricio et al. 2018). Also, their similarity to the nanostructured nature of the tissue microenvironment suggests them as a promising option for tissue engineering. Many evidences have shown that the interaction between MSCs and biomaterials could introduce a great therapeutic modality for tissue repair and regeneration since this can affect the fate and function of stem cells (Huang et al. 2012). Moreover, it has been shown that culturing BM-MSCs with a scaffolding material, like hydroxyapatite, augments bone formation after subcutaneous implantation of BM-MSCs in vivo (Akahane et al. 2012).

Many studies showed that bioactive nanomaterials have a favorable osteoinductive activity on MSCs. For example, a recent report of Niu et al. (2019) suggested that molybdenum-doped bioactive glass nanoparticles enhance both the osteogenic and mineralization ability of adipose derived stem cells. Also, a study of Wang et al. (2017b) reported that magnetic iron oxide nanoparticles induce the osteogenic differentiation of MSCs via up-regulating the long non-coding RNA INZEB2 necessary for maintaining the osteogenesis of MSCs. Moreover, Naruphontjirakul et al. (2019) indicated that spherical strontium containing bioactive glass nanoparticles are not cytotoxic and stimulate the osteogenic differentiation of human BM-MSCs. However, a study of Nguyen et al. (2019) reported that BMMSCs treated with subcytotoxic concentrations of silver nanoparticles $(5 \mu \mathrm{g} / \mathrm{mL}, 10 \mu \mathrm{g} / \mathrm{mL})$, obtained from 24-h exposure assay, reduced cell proliferation and osteogenic differentiation markers expression after 21-day culture.

Nano-hydroxyapatite (HA-NPs), a derivative of Calcium Phosphate crystals, has been shown to be chemically and structurally similar to that of the natural bone tissue (Liu et al. 2010a; Eliaz and Metoki 2017). Moreover, its excellent properties such as 
bioactivity, biocompatibility and osteoconductivity made it a promising material for many biomedical purposes, either for dental application or as bone substitute (Sobczak-Kupiec et al. 2014). Nano-hydroxyapatite-coated tantalum scaffolds have been found to increase new bone formation 2 weeks after being implanted into rat calvarial bone when compared to micron-sized hydroxyapatite-coated scaffolds (Lock and Liu 2011).

Gold nanoparticles (Au-NPs) have been extensively used in a variety of medical employments like drug delivery and imaging, owing to their unique properties, including low toxicity, colloidal stability, biocompatibility and surface modification versatility as compared to other types of nanoparticles (Singh et al. 2018). Au-NPs have been used in regenerative medicine such as cartilage and bone repair (Zhang et al. 2014a). Moreover, it has been previously reported that citrate-reduced Au-NPs can promote the osteogenic differentiation of BM-MSCs via mitogen-activated protein kinase (MAPK) signaling axis. Similarly, Au-NPs-hydrogel complex has been shown to stimulate the osteogenic differentiation of fibroblasts through the bone morphogenetic protein (BMP) signaling module (Heo et al. 2014).

Chitosan, a deacetylated derivative of chitin, is composed of Glucosamine and $\mathrm{N}$-acetylglucosamine. It is a natural biopolymer found in crustacean shells with a structural similarity to glycosaminoglycan found in bone (Levengood and Zhang 2014). The outstanding properties of chitosan, including osteoconductivity, biocompatibility, bioactivity and biodegradability, render it the most attractive natural biopolymer for biomedical purposes as an alternative to collagen in bone tissue engineering (RodríguezVázquez et al. 2015).

Based on the aforementioned data, the current work was tailored to assess the potential effect of some selected nanoparticles (HA-NPs, Au-NPs, C-NPs, Au/ HA-NPs and CH-NPs) as osteoinductive materials on BM-MSCs in vitro. This goal was achieved through gene expression analysis of osteoblast-specific genes and bone matrix mineralization detection. Also, this study sought to determine which of the studied nanoparticles could exert the most osteogenic influence on BM-MSCs to be applied for bone tissue engineering.

\section{Materials and methods}

Nanomaterials

Gold nanoparticles (Au-NPs) (CAS no. 7440-57-5) were supplied from Sigma-Aldrich Co., USA, whereas hydroxyapatite nanoparticles (HA-NPs), chitosan nanoparticles (C-NPs), gold/hydroxyapatite (Au/HANPs), and chitosan/hydroxyapatite (CH-NPs) nanoplatforms were purchased from Nano Tech. Co., 6th October City, Egypt. In brief, HA-NPs were prepared by wet chemical method as previously described by Yubao et al. (1993), while C-NPs were prepared by ionotropic gelation process according to Grenha et al. (2005). CH-NPs were obtained by gently mixing the HA-NPs powder with the chitosan solution in a final weight ratio of 1:1, while Au/HA-NPs were prepared by mechanical mix of Au-NPs and HA-NPs (1:1).

\section{Characterization of nanomaterials}

Transmission electron microscope (TEM) analysis of nanoparticles All nanoparticles were characterized using JEOL JEM-2100 high-resolution transmission electron microscope (TEM) at an accelerating voltage of $200 \mathrm{kV}$ to determine their particle size and shape. Samples were prepared by dispersing the nanomaterials in phosphate buffer saline (PBS), except chitosan nanoparticles (dispersed in dimethyl sulfoxide, DMSO), and sonication using ultrasonic water bath (Grant XUBA1, UK) for $10 \mathrm{~min}$, followed by applying one drop of each of these suspensions on copper grids coated with a thin film of carbon.

Zeta potential analysis Zeta potentials of all nanomaterials were measured using a Zetasizer Nano ZS (Malvern Instrument, UK) equipped with a $633 \mathrm{~nm}$ laser. The reference standard (DTS1230, zetapotential standard from Malvern) was used to qualify the performance of the instrument. In brief, samples (2 mg) were suspended in $1 \mathrm{ml}$ of PBS, sonicated for 15 min at $40{ }^{\circ} \mathrm{C}$ using ultrasonic water bath (Grant XUBA1, UK) to assure good dispersion, followed by vortex for $1 \mathrm{~min}$ and then filtered using a $0.22 \mu \mathrm{m}$ filter before measurement. Each of the prepared samples was dispensed into a clean disposable capillary cell (DTS1060, Malvern) which was flushed before every re-filling. Malvern Instrument's 
Dispersion Technology software (Version 4.0) was used for data analysis and zeta-potential values were estimated from the measured electrophoretic mobility data using Smoluchowski equation (Hunter 1981).

Mesenchymal stem cells (MSCs)

\section{Isolation and cultivation of BM-MSCs}

Bone marrow cells were harvested by flushing the tibia and femur bones of eight week-old male albino rats of Wistar strain weighing 100-120 g (procured from the Animal facility of the National Research Centre, Giza, Egypt) with PBS supplemented with $1 \%$ penicillinstreptomycin (Biowest, France) under aseptic condition. After centrifugation, the cell pellet was cultured in $25 \mathrm{~cm}^{2}$ flasks containing high glucose Dulbecco's modified Eagle's medium (HG-DMEM, Biowest, France) supplemented with $30 \%$ fetal bovine serum (Biowest, France) and 1\% penicillin-streptomycin. Cells were then incubated at $37{ }^{\circ} \mathrm{C}$ in $5 \%$ humidified $\mathrm{CO}_{2}$ for 2 days and non-adherent cells were removed by changing the culture medium. Culture media were replaced every 2-3 days for 12 days or till developing 90\% cell confluence. BM-MSCs, as primary culture, were then sub-cultured by enzymatic digestion using $0.25 \%$ trypsin/EDTA (Biowest, France) for $5 \mathrm{~min}$ at $37{ }^{\circ} \mathrm{C}$. BM-MSCs cultures were propagated, till reaching the third passage to ensure that all cells are morphologically homogeneous.

The protocol of this study was conducted in accordance with the ethical considerations for the experimental animals and approved by the Ethical Committee of Medical Research at the National Research Centre, Giza, Egypt, which follows the recommendations of the National Institutes of Health Guide for the care and use of laboratory animals (Approval code. 15106).

\section{BM-MSCs identification}

Morphology of BM-MSCs BM-MSCs identity was confirmed by their plastic-adherent ability and their spindle-shaped morphology through inverted microscope examination.

Flow cytometry analysis Flow cytometry analysis for cluster of differentiation (CD) 34, CD45, CD73, CD90 and CD105 cell surface markers was performed to ensure that the isolated BM-MSCs maintain their phenotype even after propagation in culture (Woodbury et al. 2000). In brief, BM-MSCs of third passage were released from culture flasks using trypsin/EDTA digestion, suspended in PBS containing $0.5 \%$ bovine serum albumin (BSA)/ $2 \mathrm{mmol}$ EDTA and analyzed for surface markers expression using anti-rat phycoerythrin (PE)conjugated CD73 and anti-rat PE-conjugated CD90 antibody (R\&D Systems,UK), anti-rat fluorescein isothiocyanate (FITC)-labeled CD105 antibody (MiltenyiBiotec, Germany), anti-rat FITC-labeled CD45 antibody and anti-rat PE-conjugated CD34 antibody (Beckman Coulter Co.,USA). Then, the cells were rinsed twice with PBS supplemented with $2 \%$ BSA, re-suspended in PBS followed by flow cytometry analysis using Beckman Coulter Elite XL, USA instrument. Isotype control matched rat immunoglobulins was employed for autofluorescence.

Tri-lineage differentiation of BM-MSCs BM-MSCs identity was further confirmed by their ability to differentiate into three different mesodermal lineages (adipogenic, chondrogenic and osteogenic) using StemPro ${ }^{\circledR}$ differentiation medium according to the manufacturer's manual.

\section{- Adipogenic differentiation of BM-MSCs}

Briefly, BM-MSCs of 3rd passage were seeded in 6-well culture plate at a density of $5 \times 10^{5}$ cells/well and then incubated at $37{ }^{\circ} \mathrm{C}$ to allow cells to expand till reaching $90 \%$ confluence. After that, adipogenic differentiation of BM-MSCs were induced by replacing the growth media with StemPro ${ }^{\circledR}$ adipogenesis differentiation medium (Cat. \#A1007001, Gibco, Thermo Fisher Scientific Inc., USA). Wells, containing cells suspended in culture medium consisted of HG-DMEM supplemented with 10\% FBS and 1\% penicillin/streptomycin, was assigned as a control. Cells were incubated at $37{ }^{\circ} \mathrm{C}$ in a humidified atmosphere containing $5 \% \mathrm{CO}_{2}$ and the media were changed every 3 days for 17 days. Following the adipogenic differentiation, both undifferentiated and differentiated cells were subjected to fixation by adding $4 \%$ formaldehyde for $30 \mathrm{~min}$, washed twice with PBS and then stained with $0.2 \%$ oil red O working solution (Sigma-Aldrich, St. Louis, MO, USA) and incubated for $20 \mathrm{~min}$ at room temperature in 
dark. Finally, cells were visualized under the inverted microscope (Olympus, Japan) to detect the intracellular accumulation of lipid vesicles appearing as bright red spheres.

\section{- Chondrogenic differentiation of BM-MSCs}

In brief, BM-MSCs of third passage were seeded into 6-well culture plate at a density of $5 \times 10^{5}$ cells/ well and then incubated at $37{ }^{\circ} \mathrm{C}$ to allow cells to expand till reaching $90 \%$ confluence. After that, BMMSCs were induced for chondrogenic differentiation by replacing the growth media with $\mathrm{StemPro}{ }^{\circledR}$ chondrogenesis differentiation medium (Cat. \# A1007101, Gibco, Thermo Fisher Scientific Inc., USA). Control wells represented cells suspended in culture medium consisted of HG-DMEM supplemented with 10\% FBS and $1 \%$ penicillin/streptomycin. Cells were then incubated at $37{ }^{\circ} \mathrm{C}$ with $5 \%$ humidified $\mathrm{CO}_{2}$ and the media were replaced every 3 days for 17 days. Following the chondrogenic differentiation, both undifferentiated and differentiated cells were fixed with $4 \%$ formaldehyde for $30 \mathrm{~min}$. After fixation, cells were washed twice with PBS and then stained with $1 \%$ alcian blue working solution (Sigma-Aldrich, St. Louis, MO, USA) prepared with $3 \%$ glacial acetic acid and incubated for $20 \mathrm{~min}$ at room temperature in the dark. Finally, cells were visualized under the inverted microscope to detect the formation of glycosaminoglycan stained in blue color.

\section{- Osteogenic differentiation of BM-MSCs}

BM-MSCs were induced to differentiate into osteocytes using StemPro ${ }^{\circledR}$ osteogenesis differentiation medium purchased from Gibco, Thermo Fisher Scientific Inc. (USA) (Cat. \# A1007201). Control wells contained cells suspended in culture medium consisted of HG-DMEM supplemented with 10\% FBS and $1 \%$ penicillin/streptomycin. After 21 days of differentiation, both undifferentiated and differentiated cells were fixed with $4 \%$ formaldehyde solution for $30 \mathrm{~min}$. After fixation, the wells were rinsed with PBS and the cells were stained with $2 \%$ alizarin red S solution ( $\mathrm{pH}$ 4.2) (Sigma-Aldrich, St. Louis, MO, USA) for $20 \mathrm{~min}$. Then, the cells were visualized under the inverted microscope to detect the formation of mineralized nodules of the extracellular matrix.
In vitro study

\section{MTT assay}

Influence of the tested nanoparticles on rat BM-MSCs viability was quantified by using 3-[4,5-dimethylthiazol-2-yl]-2,5 diphenyl tetrazolium bromide (MTT) assay (SERVA Electrophoresis GmbH Heidelberg, Germany, CAS No.298-93-1), as previously described by Van Meerloo et al. (2011). In Brief, BM-MSCs of third passage were seeded into 96-well tissue culture plates at a density of $1 \times 10^{4}$ cells/well and incubated at $37{ }^{\circ} \mathrm{C}$ (in $5 \% \mathrm{CO}_{2}$ and $90 \%$ humidity) for $24 \mathrm{~h}$. Then, each of the studied nanomaterials at different concentrations $(2,5,10,15,20$ and $25 \mu \mathrm{g} / \mathrm{ml})$ was added into their specific wells (treated wells) in triplicate. Control wells represented cells treated with vehicle (DMSO in case of C-NPs and $0.1 \%$ ascorbic acid in case of $\mathrm{CH}-\mathrm{NPs}$ ) or left untreated, whereas blank wells referred to that containing culture medium only (without cells). After $48 \mathrm{~h}$ and $72 \mathrm{~h}$ incubation period, the cells were washed with PBS and then MTT solution (dissolved in serum-containing media) of $0.5 \mathrm{mg} / \mathrm{ml}$ concentration was added to each well followed by incubating the plates at $37^{\circ} \mathrm{C}$ for $4 \mathrm{~h}$. After incubation, formazan crystals, formed upon reduction of MTT by metabolically active cells, were then dissolved by DMSO. The optical density (OD) of each well was measured at $492 \mathrm{~nm}$ and a reference wavelength of $630 \mathrm{~nm}$, using a microplate reader (Model 4300; Chromate Instrument, Awareness technology, Inc., Palm City, USA). The percentage of cell viability compared with the control was calculated according to the following equation:

Percentage of cell viability $=\frac{\mathrm{ODt}-\mathrm{ODb}}{\mathrm{ODc}-\mathrm{ODb}} \times 100$

where ODt is the mean value of the measured OD of the treated wells, ODc is the mean value of the measured OD of the control wells, ODb is the mean value of the measured OD of the blank wells.

\section{Differentiation of BM-MSCs into osteoblasts}

BM-MSCs cultures of the third passage $(90 \%$ cell confluence) were induced to differentiate into osteoblasts upon culturing in osteogenic medium (OS) containing $5.0 \mathrm{mM} \beta$-glycerophosphate, $50 \mu \mathrm{g} / \mathrm{ml}$ ascorbic acid and $0.1 \mu \mathrm{M}$ dexamethasone (all 
purchased from Sigma-Aldrich, St. Louis, MO, USA) (Yi et al. 2010), in combination with one of the tested nanomaterials for 14 days at a concentration selected based on MTT results.

Gene expression analysis

\section{$R N A$ extraction and $q R T-P C R$}

Following the differentiation of BM-MSCs into osteoblasts using OS medium in the presence or absence of the selected nanoparticles for 7 days, total RNA was extracted from undifferentiated and differentiated BM-MSCs cultures (osteoblasts) using the RNeasy mini kit for total RNA purification from animal cells (Cat. \#74104, Qiagen, Germany), according to the manufacturer's protocol. RNA concentration was determined in duplicate using Nano Drop 2000 (Thermo Fisher Scientific, Rockford, IL, USA) and 260/280 nm ratio was assessed to ensure RNA purity. RNA samples were pre-treated with RNasefree DNase I (1U/ $\mu$ l) (Cat \# EN0521, Thermo Fisher Scientific Inc., USA) before being reverse transcribed into cDNA, using Revert Aid first strand cDNA synthesis kit (Cat\# K1621, Thermo Fisher Scientific, Inc., Lithuania) according to the manufacturer's instructions. The expression levels of Runt-related transcription factor 2 (Runx-2) and bone morphogenetic protein 2 (BMP-2) genes were determined by quantitative real-time PCR (qRT-PCR) using QuantiTect SYBR Green PCR Kit (Cat\# 204141, Qiagen, Germany) and 10X Quantitect Primer Assays (Qiagen, Germany): Runx-2 (Cat\# QT01620647), BMP-2 genes (Cat\# QT00495096) and $\beta$-actin (Cat\# QT00193473). The StepOne Real Time PCR instrument (Applied Biosystems, USA) was used for realtime analysis. The reaction mixture contained $12.5 \mu \mathrm{l}$ SYBR master mix, $2.5 \mu$ primer assay (1X), cDNA template $(100 \mathrm{ng})$ and nuclease-free water, in a final volume of $25 \mu \mathrm{l}$. The thermal conditions were adjusted as follows: initial denaturation step at $95{ }^{\circ} \mathrm{C}$ for $15 \mathrm{~min}$, followed by 40 repetitive cycles of denaturation at $94{ }^{\circ} \mathrm{C}$ for $15 \mathrm{~s}$, annealing at $55^{\circ} \mathrm{C}$ for $30 \mathrm{~s}$ and extension at $72{ }^{\circ} \mathrm{C}$ for $30 \mathrm{~s}$, as well as a final extension step at $72{ }^{\circ} \mathrm{C}$ for $5 \mathrm{~min}$. The quantification of the relative mRNA expression was determined using the $2^{-\Delta \Delta \mathrm{Ct}}$ method (Livak and Schmittgen 2001).
Alizarin red S staining

BM-MSCs were seeded into 6-well tissue culture plates at a density of $5 \times 10^{5}$ cells/well and incubated at $37{ }^{\circ} \mathrm{C}$ in $5 \% \mathrm{CO}_{2}$ humidified incubator till forming $90 \%$ confluent cell sheet. The media were then replaced by either OS medium alone or combined with one of the selected nanomaterials for 21 days. The formation of mineralized matrix nodules was detected by alizarin red S staining (Sigma-Aldrich, St. Louis, MO, USA). In brief, the cells were washed twice with PBS and then fixed in $4 \%$ buffered formaldehyde for $15 \mathrm{~min}$ at room temperature. After that, the cells were washed three times with distilled water, followed by staining with $1 \%$ alizarin red $\mathrm{S}$ solution ( $\mathrm{pH} 4$.2) for $20 \mathrm{~min}$ at room temperature. The alizarin red $\mathrm{S}$ solution was aspirated from wells followed by rinsing cells twice with distilled water. PBS was added to each well to avoid cell dryness. Orange-red staining indicates the formation of mineralized matrix nodules that were visualized through the inverted microscope (Huang et al. 2009).

\section{Statistical analysis}

All data were represented as mean \pm standard deviation (SD). Statistical analysis was carried out by twoway analysis of variance (ANOVA) using GraphPad Prism 7 software (La Jolla, CA, USA) and the Turkey's multiple comparisons test was employed to compare the significance between groups. The significance level was set at $P<0.05$.

\section{Results}

Characterization of nanomaterials

\section{TEM analysis results}

High-resolution transmission electron microscopy was employed to characterize the morphology and size distribution of the selected nanomaterials. TEM imaging of HA-NPs showed that they have a rod shape with a size range from 80 to $125.4 \mathrm{~nm}$ in length and 35 to $45.7 \mathrm{~nm}$ in width. Meanwhile, that of Au-NPs indicated that they have spherical shape with diameter ranged between 30.2 and $40.7 \mathrm{~nm}$. Also, TEM analysis of C-NPs revealed that they are spherical in shape 

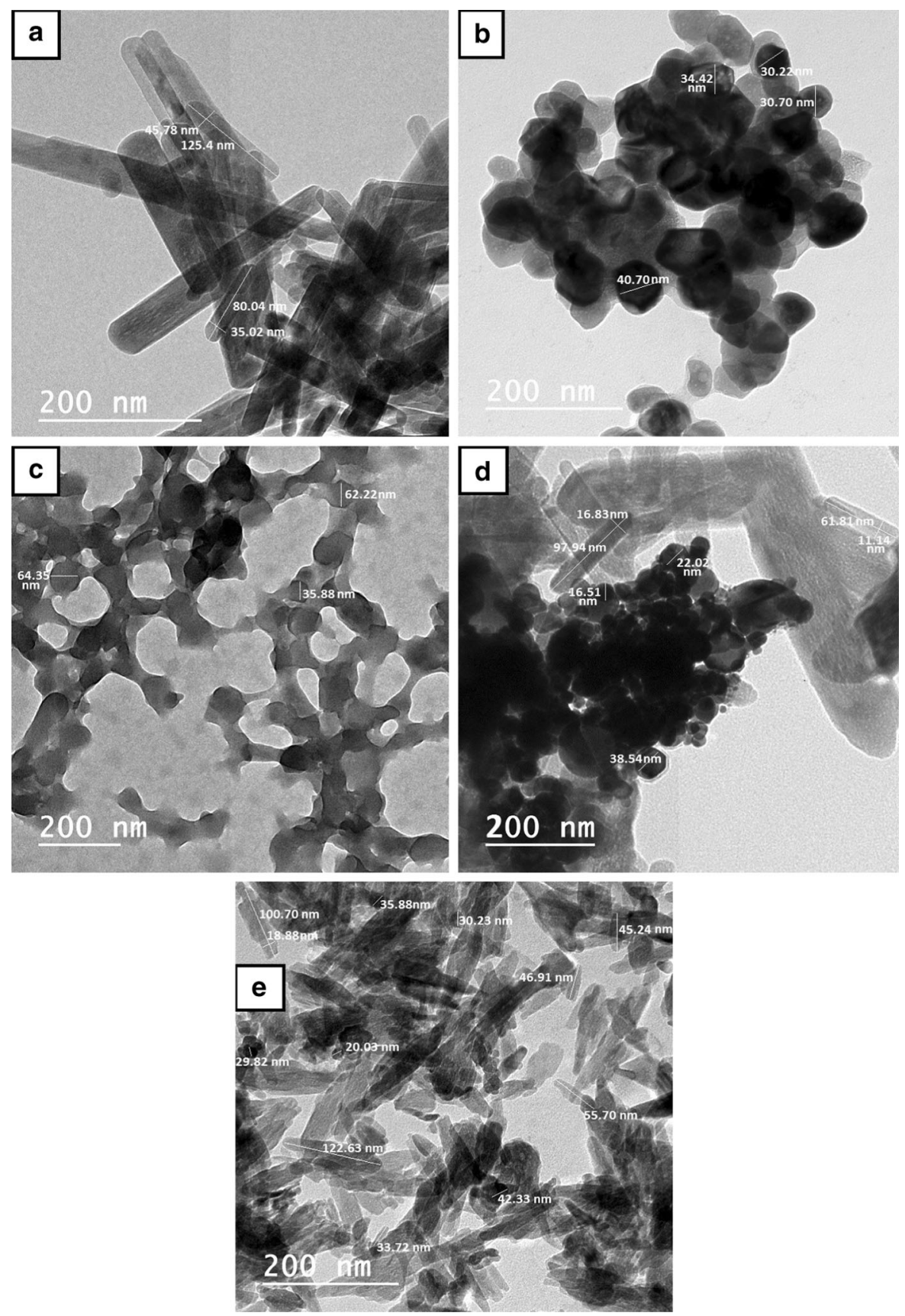

Fig. 1 High-resolution transmission electron microscopy images of: a HA-NPs, b Au-NPs, c C-NPs, d Au/HA-NPs and e CH-NPs showing the particles size and shape

with a size range of $35.8-64.3 \mathrm{~nm}$. Lastly, TEM analysis of $\mathrm{Au} / \mathrm{HA}$ and $\mathrm{CH}$ nanostructures denoted that both Au-NPs and C-NPs were successfully adsorbed on HA-NPs surface forming nanoplatforms as shown in Fig. 1.

\section{Zeta potential findings}

Malvern Zetasizer system measurements indorsed that the representative zeta potential of HA-NPs is $(-3.18 \mathrm{mV}), \quad(-29.3 \mathrm{mV})$ for Au-NPs, $(-21.3 \mathrm{mV})$ for $\mathrm{Au} / \mathrm{HA}-\mathrm{NPs},(34.8 \mathrm{mV})$ for C-NPs 
and $(-31.5 \mathrm{mV})$ for $\mathrm{CH}-\mathrm{NPs}$ as illustrated in Table 1.

\section{Characterization of BM-MSCs}

\section{Microscopic features and photodocumentation}

Inverted microscope examination displayed plasticadherent spindle-shaped BM-MSCs in colonies, as shown in Fig. 2, which confirms the identity of BMMSCs.

\section{Flow cytometry analysis}

Flow cytometry analysis showed that isolated BMMSCs were positive for CD73 (89.8\%), CD105 (85.7\%), CD90 (83.5\%), and negative for CD34 (8.1\%) and CD 45 (0.6\%) (Fig. 3).

Table 1 Representative zeta potential stability of the selected nanostructures using Malvern ZetaSizer system

\begin{tabular}{lc}
\hline Nanostructure & Zeta potential value $(\mathrm{mV})$ \\
\hline HA-NPs & -3.18 \\
Au-NPs & -29.3 \\
Au/HA-NPs & -21.3 \\
C-NPs & 34.8 \\
CH-NPs & -31.5 \\
\hline
\end{tabular}

\section{Tri-lineage differentiation of BM-MSCs}

BM-MSCs were successfully induced to differentiate into adipocytes upon culturing in adipogenic differentiation media. The synthesis of intracellular lipid droplets in adipocytes was confirmed by staining with oil red O (Fig. 4a). Also, BM-MSCs were differentiated into chondrocytes which was confirmed by alcian blue staining (Fig. 4b). Moreover, osteogenic differentiation of BM-MSCs was documented by alizarin red S staining as shown in Fig. 4c.

In vitro study data

\section{The influence of nanomaterials on BM-MSCs viability}

The cytotoxic effect of the tested nanomaterials on BM-MSCs viability was assessed with six different concentrations $(2,5,10,15,20$ and $25 \mu \mathrm{g} / \mathrm{ml})$ at $48 \mathrm{~h}$ and $72 \mathrm{~h}$ using MTT assay.

It was noticed that all studied nanomaterials displayed insignificant toxicity on BM-MSCs at the suggested concentrations after $48 \mathrm{~h}$ incubation except for CH-NPs and C-NPs at certain concentrations. With further extension of the incubation time to $72 \mathrm{~h}$, all nanomaterials still exhibited negligible cytotoxic impact even at the highest concentration of $25 \mu \mathrm{g} /$ $\mathrm{ml}$, with the exception of C-NPs (Fig. 5e).

The percent of BM-MSCs viability after being exposed to $5 \mu \mathrm{g} / \mathrm{ml}$ Au-NPs was $99.9 \pm 0.68$ and $110.6 \pm 7.6 \%$ after $48 \mathrm{~h}$ and $72 \mathrm{~h}$ incubation, respectively, with no significant difference from control

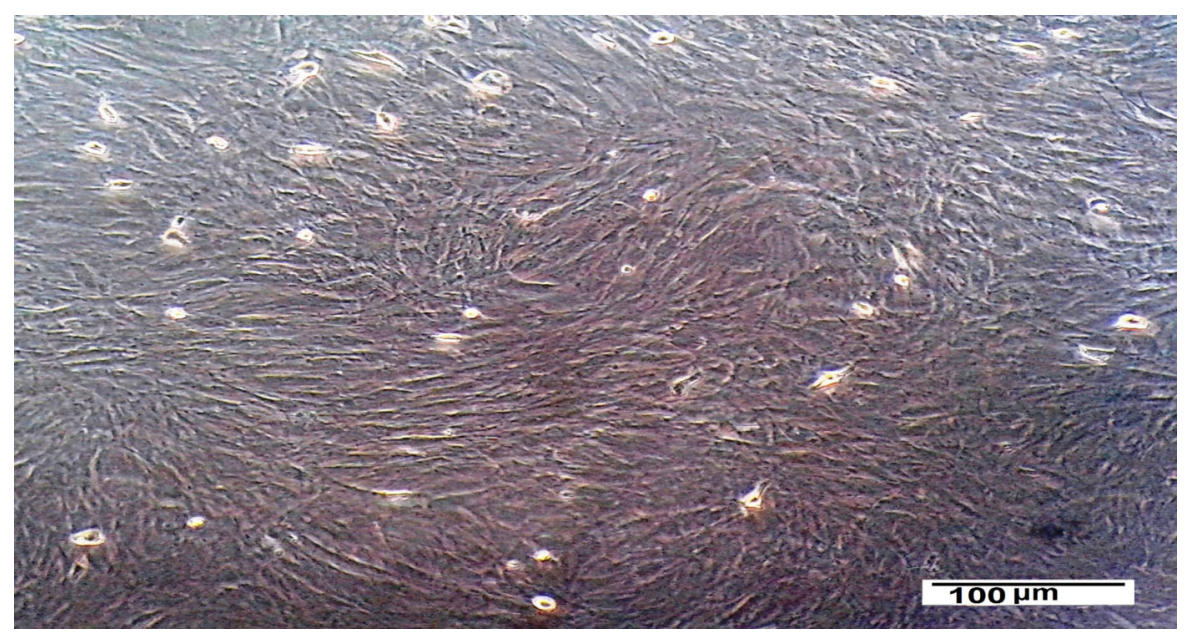

Fig. 2 Microscopic features of rat BM-MSCs (passage 3) in culture flasks showing confluent cell sheet of spindle-shaped morphology 
Fig. 3 Flow cytometry analysis of BM-MSCs a cluster of differentiation (CD) 73 (89.8\%), b CD105 (85.7\%), c CD90 (83.5\%), d CD34 (8.1\%) and e CD45 $(0.6 \%)$, antibodies; where CD73, CD105 and CD90 stand for the fluorescenttagged antibodies used to detect the surface antigens that are positively expressed on mesenchymal stem cells (MSCs), while CD34 and CD45 stand for the fluorescent-tagged antibodies used to detect the cell surface antigens that are only expressed on the hematopoietic stem cells and are negatively expressed on MSCs. The horizontal line in each panel represents the channel in the flow cytometry that detects the fluorescent-tagged antibodies used for detection of the expression of these markers on the MSCs
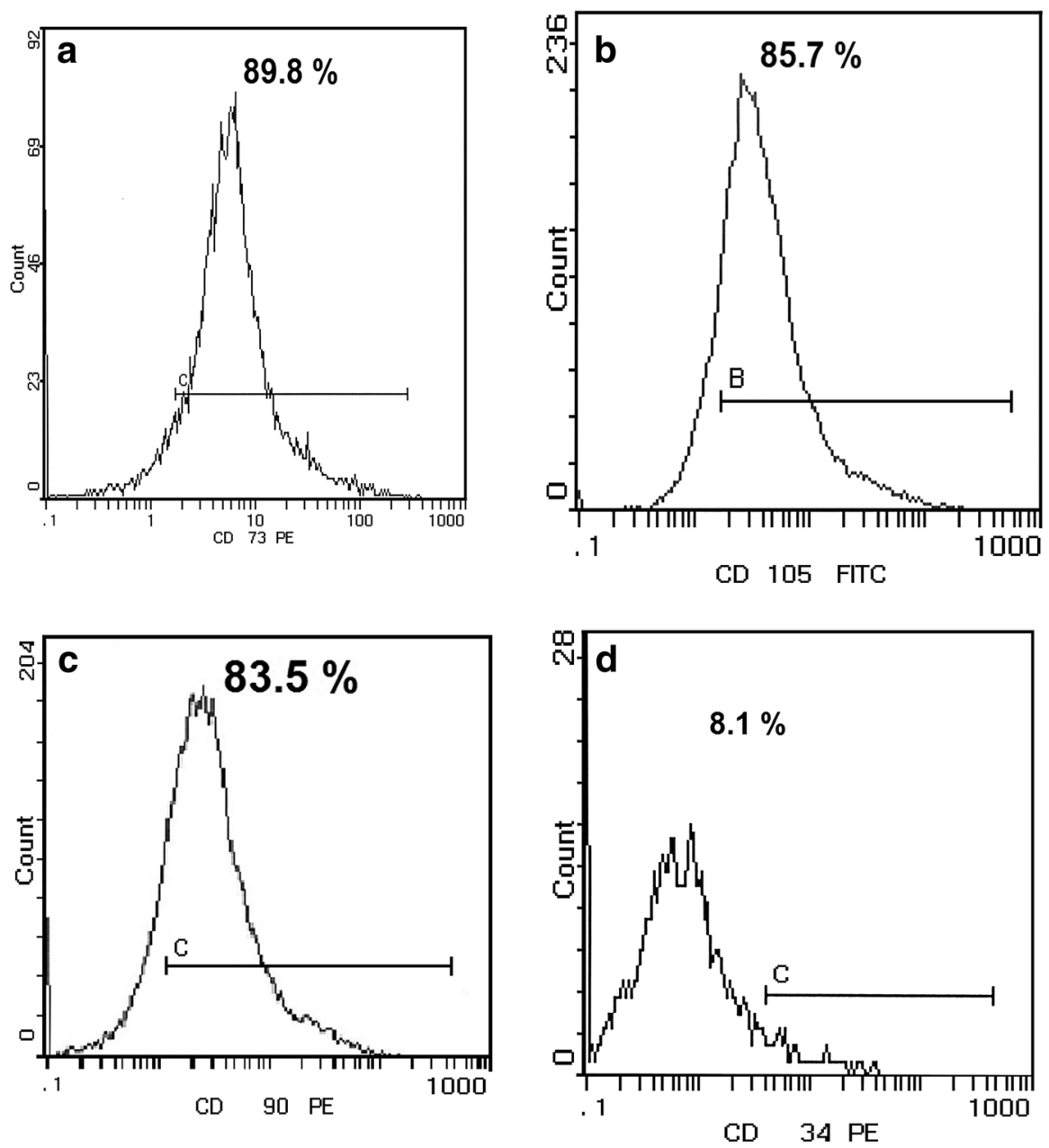

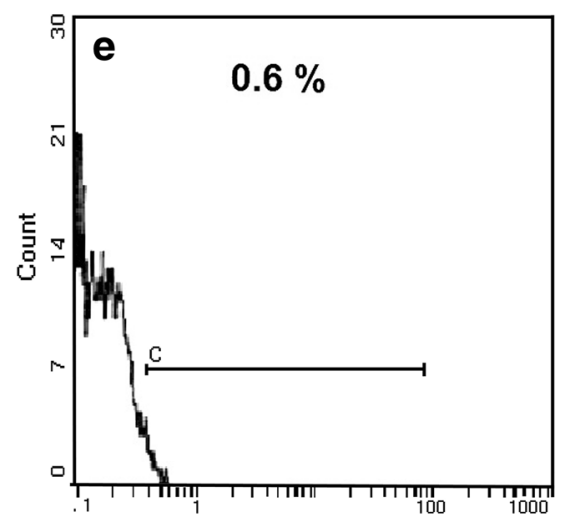

$(P>0.05)$. Similarly, the viability of BM-MSCs after treatment with $5 \mu \mathrm{g} / \mathrm{ml} \mathrm{Au/HA-NPs} \mathrm{was} 109.7 \pm 7.4$ and $102.02 \pm 14.1 \%$ after $48 \mathrm{~h}$ and $72 \mathrm{~h}$ incubation, respectively, showing insignificant difference $(P>0.05)$ from control. Also, $5 \mu \mathrm{g} / \mathrm{ml}$ concentration of CH-NPs revealed insignificant cytotoxicity $(P>0.05)$ as compared to control and BM-MSCs viability was found to be $91.5 \pm 1.6$ and $103.02 \pm 5.6 \%$ after $48 \mathrm{~h}$ and $72 \mathrm{~h}$, respectively. Whereas, BM-MSCs displayed a percent of viability 

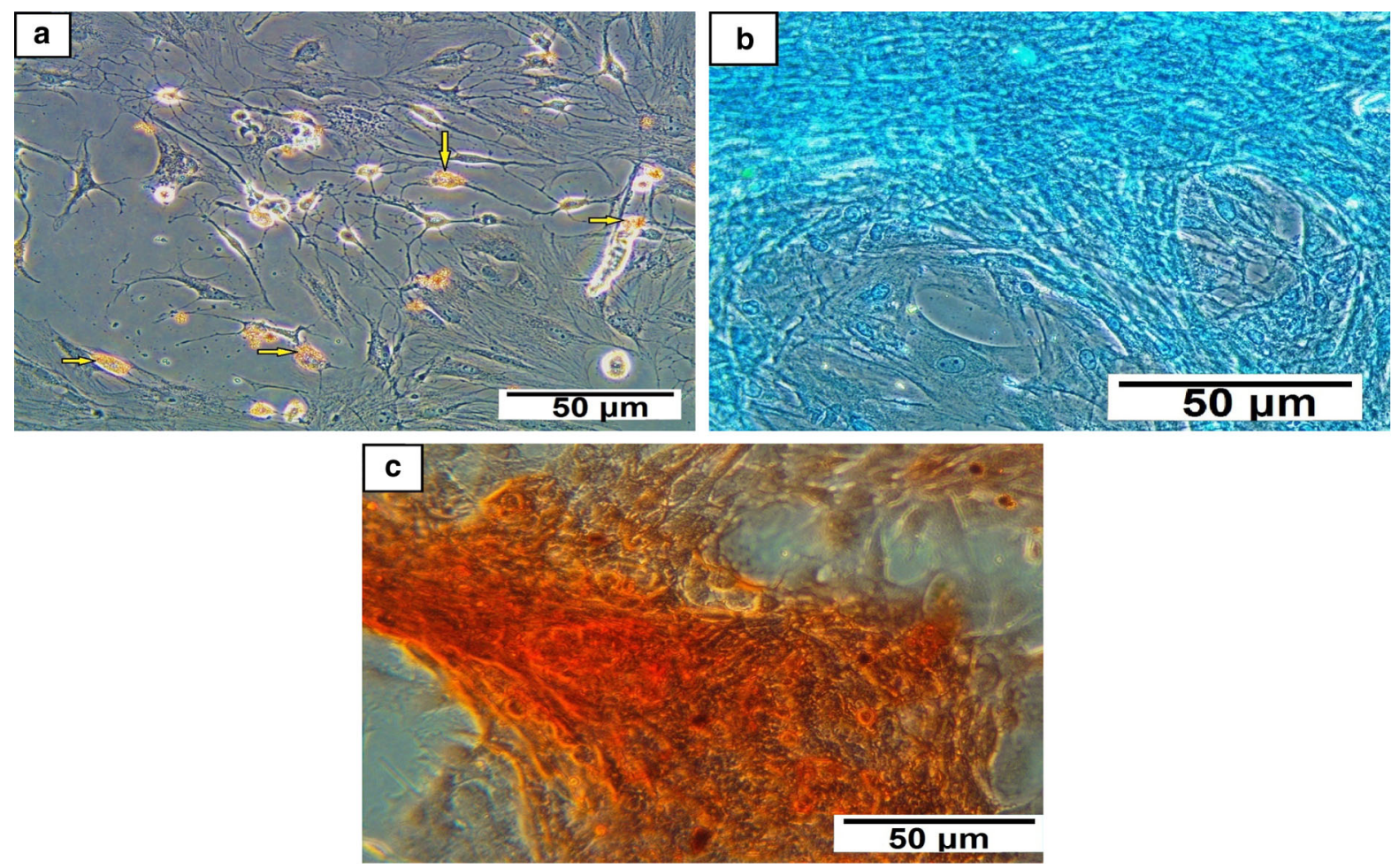

Fig. 4 Photomicrographs of BM-MSCs differentiated into: a adipocytes (yellow block arrows refer to intracellular lipid droplets) stained with oil red $\mathrm{O}$ stain, $\mathbf{b}$ chondrocytes showing the blue-stained proteoglycan using alcian blue stain, and

of $112.2 \pm 4.2$ and $116.5 \pm 14.8 \%$ after being treated with $10 \mu \mathrm{g} / \mathrm{ml}$ of HA-NPs for $48 \mathrm{~h}$ and $72 \mathrm{~h}$, respectively, indicating insignificant change $(P>0.05)$ as compared to control. For C-NPs, $5 \mu \mathrm{g} / \mathrm{ml}$ concentrations resulted in a percentage cell viability of $111.9 \pm 4.3$ and $109.6 \pm 10.3 \%$ after $48 \mathrm{~h}$ and $72 \mathrm{~h}$ incubation, respectively, without any significant difference from control $(P>0.05)$. Therefore, the concentration of $10 \mu \mathrm{g} / \mathrm{ml}$ for HA-NPs and $5 \mu \mathrm{g} / \mathrm{ml}$ for Au-NPs, Au/HA-NPs, CH-NPs and C-NPs were selected to be used later in all osteogenic differentiation experiments, since such selected concentrations revealed the least insignificant cytotoxic action on BM-MSCs as compared to control.

It would be pertinent to mention that BM-MSCs treated with all nanoparticles, at some studied concentrations over $48 \mathrm{~h}$ and $72 \mathrm{~h}$ incubation periods, presented slightly higher proliferation than that of the control cells (insignificant difference) as cell viability showed a slight increase (only 10-20\%) with no noticeable dose or time-dependent tendency. This c osteocytes indicating the accumulation of mineralized matrix stained in red orange color using alizarin red S stain. (Color figure online)

means that such nanoparticles have no effect on BMMSCs proliferation or the pattern of cell behavior.

Gene expression profile

The osteospecific gene expression profile for both differentiated and undifferentiated BM-MSCs assessed after 7 days of culture is represented in Fig. 6. The results indicated that the gene expression level of Runx-2 was significantly up-regulated $(P<0.05)$ to 3.1 folds upon culturing of BM-MSCs in osteogenic medium alone (BM-MSCs + OS) when compared with undifferentiated BM-MSCs (control). While culturing BM-MSCs in osteogenic medium coupled with Au-NPs or Au/HA-NPs elicited significant up-regulation $(P<0.05)$ of Runx-2 gene expression level to 4.6 and 1.8 folds, respectively, as compared to BM-MSCs + OS. However, Runx-2 gene expression level revealed insignificant overexpression $(P>0.05)$ to 1.4 and 1.2 folds upon culturing BM-MSCs in osteogenic medium combined 

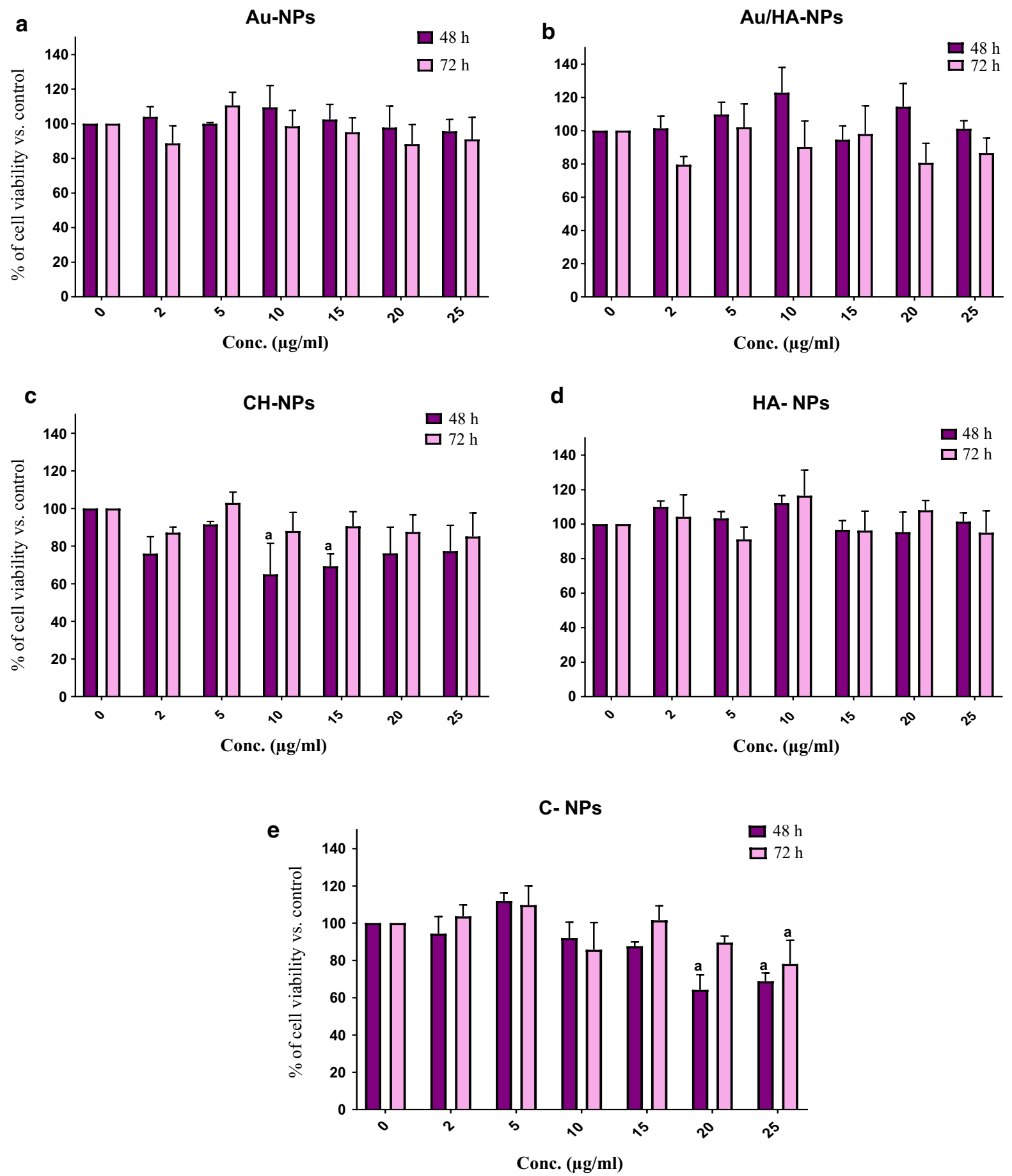

Fig. 5 Percent viability of BM-MSCs treated with the tested nanoparticles: a Au-NPs, b Au/HA-NPs, c CH-NPs, d HA-NPs and e C-NPs at different concentrations for $48 \mathrm{~h}$ and $72 \mathrm{~h}$. Data are represented as mean of percent cell growth \pm SD. Three replicates were set up for each treatment group $(n=3)$ with experiments repeated in duplicate. a Means are significantly different from the control at $P<0.05$ 


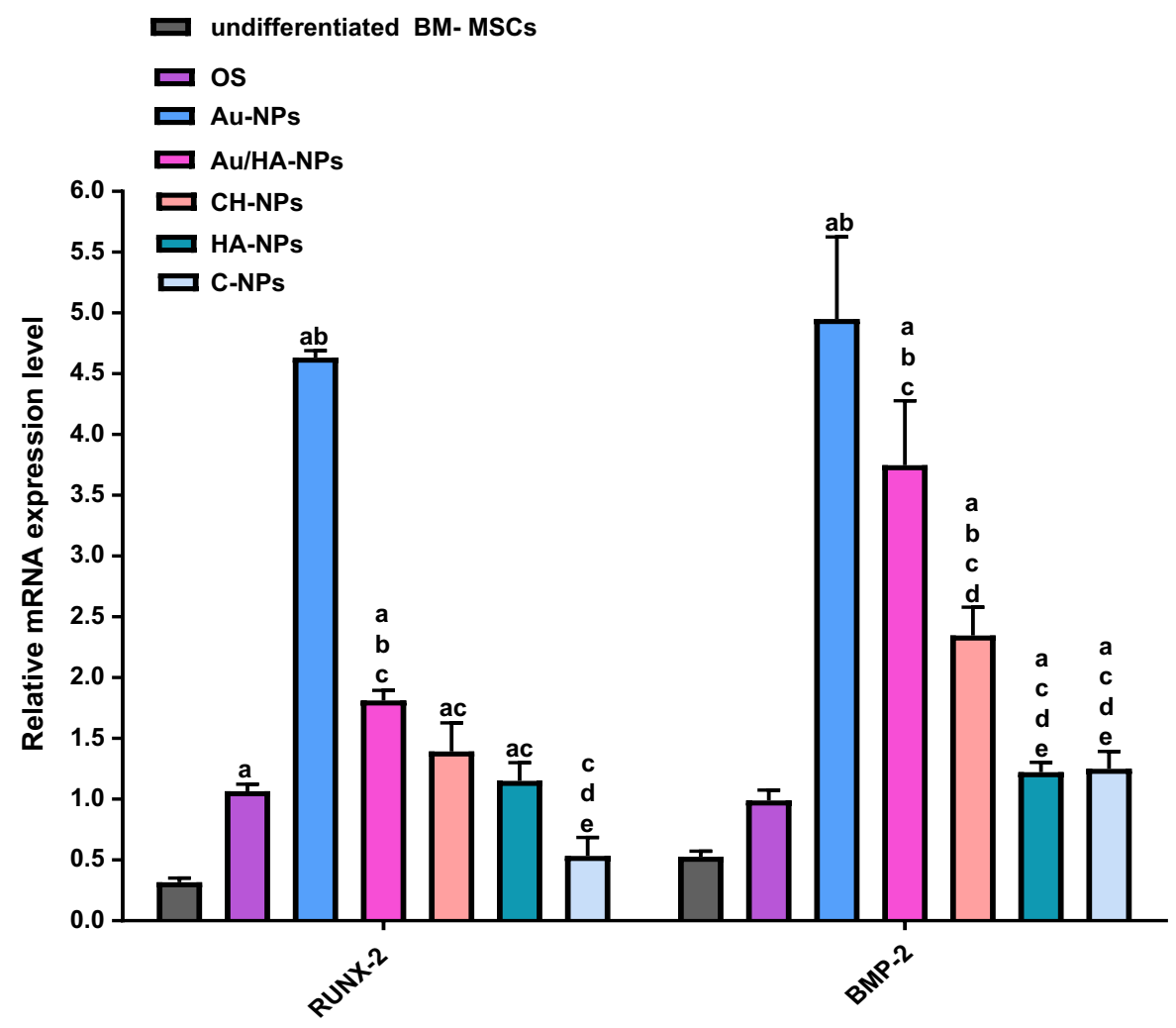

Fig. 6 Relative gene expression analysis of osteoblast-specific genes (Runx-2 and BMP-2) in both differentiated and undifferentiated BM-MSCs. Data are expressed as mean \pm SD, obtained from three independent experiments $(n=3)$. a significance difference from undifferentiated BM-MSCs at $P<0.05$, b significance difference from OS (BM-MSCs cultured in OS

with CH-NPs or HA-NPs, respectively, as opposed to BM-MSCs + OS. Also, BM-MSCs cultured in osteogenic medium supplemented with C-NPs displayed insignificant change $(P>0.05)$ in Runx-2 gene expression level by 0.5 fold versus BM-MSCs + OS. BMP-2 gene expression level exhibited insignificant amplification $(P>0.05)$ to 1.7 fold upon culturing BM-MSCs in osteogenic medium alone relative to that of control. Whereas, when BM-MSCs were cultured with Au-NPs, Au/HA-NPs or CH-NPs in the presence of osteogenic medium, the gene expression level of BMP-2 was significantly up-regulated $(P<0.05)$ by $4.9,3.7$ and 2.3 fold change, respectively, compared to that of BM-MSCs + OS. On the other side, culturing BM-MSCs in osteogenic medium in combination with HA-NPs or C-NPs evoked insignificant up-regulation $(P>0.05)$ of BMP-2 gene expression level to 1.2 and medium alone) at $P<0.05$, c significance difference from AuNPs (BM-MSCs cultured in OS medium containing Au-NPs) at $P<0.05$, d significance difference from Au/HA-NPs (BMMSCs cultured in OS medium containing Au/HA-NPs) at $P<0.05$ and $\mathbf{e}:$ significance difference from $\mathrm{CH}-\mathrm{NPs}$ (BMMSCs cultured in OS medium containing CH-NPs) at $P<0.05$

Fig. 7 Alizarin red S staining of a undifferentiated BM-MSCs, b differentiated BM-MSCs upon culturing in OS medium only, c differentiated BM-MSCs upon culturing in OS medium supplemented with HA-NPs, d differentiated BM-MSCs upon culturing in OS medium supplemented with Au-NPs, e differentiated BM-MSCs upon culturing in OS medium supplemented with Au/HA-NPs, $\mathbf{f}$ differentiated BM-MSCs upon culturing in OS medium supplemented with C-NPs and $\mathbf{g}$ differentiated BMMSCs upon culturing in OS medium supplemented with $\mathrm{CH}$ NPs. (Color figure online)

1.25 folds, respectively, as compared to BMMSCs + OS.

Of note, culturing BM-MSCs with Au-NPs in combination with OS medium brought about the most prominent significant up-regulation $(P<0.05)$ in both Runx-2 and BMP-2 gene expression levels in comparison with those of BM-MSCs cultured in OS medium containing Au/HA-NPs, CH-NPs, HA-NPs or 

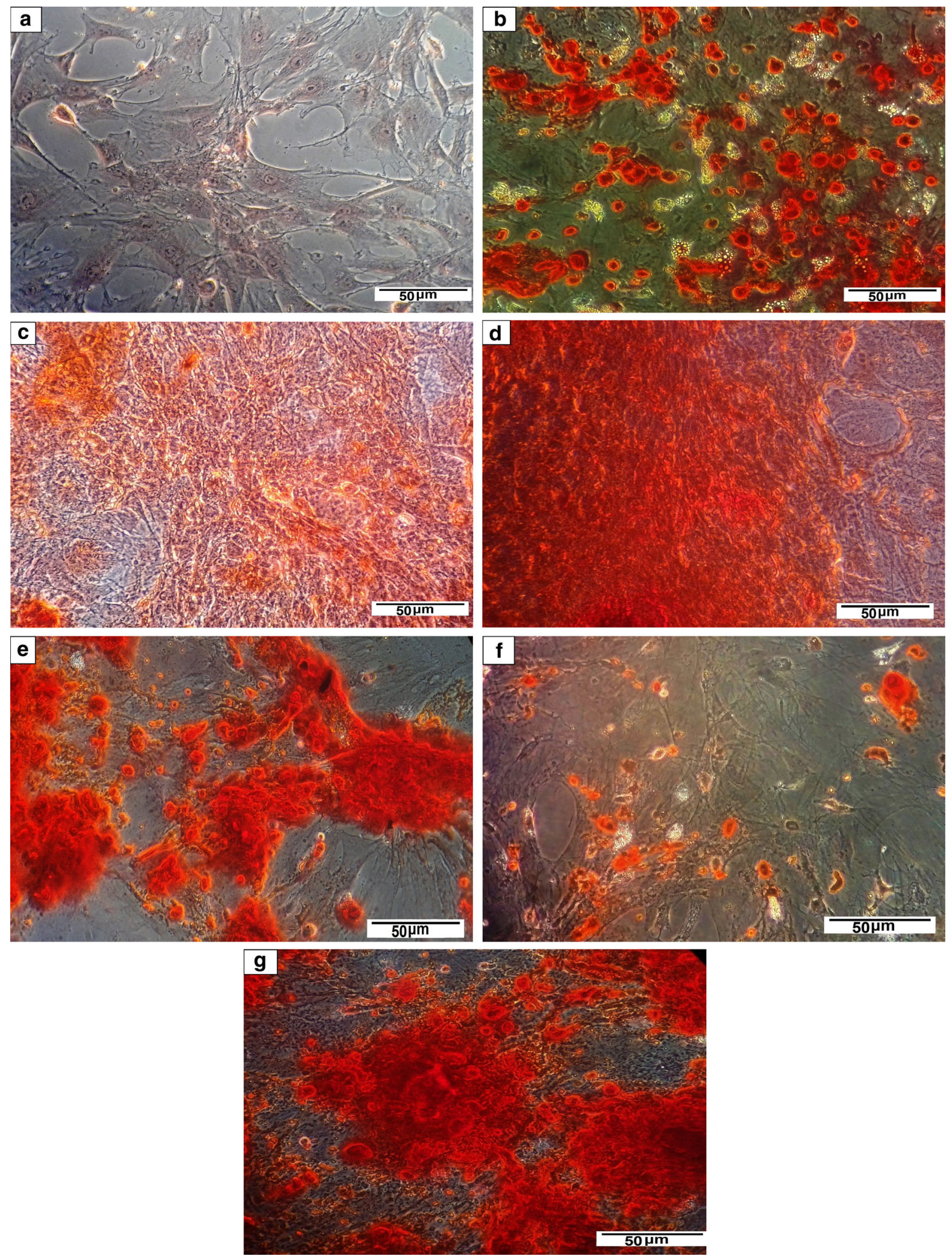
C-NPs. Also, BM-MSCs incubated with Au/HA-NPs plus OS medium yielded a significant over-expression $(P<0.05)$ of BMP-2 gene expression level compared with that cultured in OS medium supplemented with CH-NPs, HA-NPs or C-NPs. Additionally, BM-MSCs cultured in OS medium in the presence of CH-NPs provoked significant enhancement $(P<0.05)$ of BMP-2 gene expression level as compared with that cultured in OS medium containing HA-NPs or C-NPs.

The aforementioned data indicated that Au-NPs followed by $\mathrm{Au} / \mathrm{HA}-\mathrm{NPs}$ and $\mathrm{CH}-\mathrm{NPs}$ exhibited the most osteoinductive effect on BM-MSCs through upregulating Runx-2 and BMP-2 gene expression levels.

\section{Alizarin red S staining of the differentiated BM- MSCs}

The mineralized matrix nodules representing osteoblast-phenotypic markers speak for the successful osteogenic differentiation of MSCs. Therefore, the osteoinductive effect of the chosen nanoparticles on BM-MSCs was further investigated by alizarin red S staining assay. The photomicrographs in Fig. 7 revealed that the BM-MSCs were successfully motivated to generate osteoblasts upon culturing with OS medium, either alone (Fig. 7b) or in the presence of the tested nanomaterials (HA-NPs, Au-NPs, Au/HANPs, C-NPs or CH-NPs) for 21 days, as indicated by alizarin red $\mathrm{S}$ staining of calcium nodules precipitates (Fig. 7c, d, e, f, g, respectively). Meanwhile, the undifferentiated BM-MSCs represented no calcium nodules deposition (Fig. 7a). Of note, BM-MSCs morphology was observed to be changed from spindle to polygonal shape after 21 days of culture in the osteogenic medium in the presence of nanoparticles.

\section{Discussion}

Currently, MSCs have been shown to be the most prominent therapeutic options in regenerative medicine, especially in bone tissue engineering owing to their differentiation potency in addition to nonimmunogenic characteristics. Nanoplatforms have been considered as a promising alternative for implantable devices due to their ability to induce cell proliferation and differentiation with the least cell toxicity (Crisan et al. 2015). It has been stated that nanoparticles can stimulate specific signaling pathways in stem cells, resulting in their osteoinduction (Yi et al. 2010; Yang et al. 2013). Various pathways are believed to be implicated in coordinating the cellular uptake of nanoparticles, including endocytosis, phagocytosis and pinocytosis (Zhao et al. 2011).

Most of the nanoparticles used in the current study have a spherical morphology, except hydroxyapatite nanoparticles (HA-NPs), since it has been reported that spherical-shaped nanoparticles are better than their rod or star-shaped counterparts regarding cellular uptake (Freese et al. 2012a), as they induce a diminished cell toxicity by restraining the cell membrane from direct diffusion (Freese et al. 2012b). For example, the uptake of spherical gold nanoparticles (Au-NPs) by cells was found to be considerably easier compared to rod-shaped Au-NPs. Spherical Au-NPs are capable of targeting cells through active and passive means (Pissuwan et al. 2007a, b), which is likely to induce physiological changes, following the cellular interaction between the Au-NPs and target cells in vivo (Chen et al. 2013).

However, we have selected the rod shape for HANPs to mimic that of the natural bone, as we need to simulate the same natural osteogenic activity of HANPs of the native bone tissue. Since the natural bone tissue is known to be made up of $70 \%$ nanohydroxyapatite having rod-like-shaped morphology (Okada and Matsumoto 2015; Mansour et al. 2017). Moreover, a study of Lu et al. (2011) reported that rodshaped HA-NPs incorporated in poly ( $\varepsilon$-caprolactone) (PCL) film mimic the natural bone and best enable human osteoblasts to exert their biological functionality providing the right and sustainable signals for adipose tissue-derived stem cells (ASCs) osteogenic differentiation, whereas the human osteoblasts on PCL films or PCL incorporated with spherical HA-NPs lack the right phenotype and the sustainable capability for steeling osteogenic differentiation of ASCs.

In the present approach, TEM micrograph of HANPs showed relatively uniform rod shape particles with a size matching to that of nanohydroxyapatite of the natural bone microenvironment in vivo (Vandiver et al. 2005). This finding coincides with that of Zainol et al. (2008) who identified the rod-shaped hydroxyapatite nanoparticles with a size of about $40 \mathrm{~nm}$ in width and $70-100 \mathrm{~nm}$ in length upon TEM analysis. Moreover, zeta potential analysis of HA-NPs revealed negatively charged particles. This is in consistent with 
the study of Cai et al. (2007), who demonstrated negatively charged rod shaped HA-NPs with a zeta potential of $-7.1 \mathrm{mV}$.

In the present study, TEM investigation of Au-NPs revealed uniform spherical-shaped particles with negative charges indicating no particles aggregation upon dispersion. These results are in harmony with the study of $\mathrm{Li}$ and his colleagues (2016), who reported spherical-shaped gold nanoparticles with $40 \mathrm{~nm}$ size and zeta potential of $-23.15 \mathrm{mV}$.

As shown in the present attempt, TEM analysis for $\mathrm{Au} / \mathrm{HA}-\mathrm{NPs}$ was performed to confirm the adsorption of Au-NPs on the surface of HA-NPs forming nanocomposite. This observation is in agreement with that of the studies of Crisan et al. (2015) and Ferreira dos Santos et al. (2015).

The current findings indicated spherical-shaped C-NPs upon TEM investigation. This result is comparable to that of the study of Iswanti et al. (2019). Chitosan is characterized by its positive zeta potential value (Uskoković and Desai 2014). Zetasizer analysis indicated the formation of positive charges on C-NPs. This is in concert with the result of Wan et al. (2007), who indicated positively charged C-NPs of $28.5 \mathrm{mV}$. As the value of the zeta potential of the nanoparticles increases, the amount of charge on their surface increases, resulting in a strong repelling reaction between the particles providing high stability and better uniformity in size.

It is known that the incorporation of chitosan with hydroxyapatite could reduce the degree of HA-NPs aggregation, which is responsible for cell toxicity (Muller et al. 2014). TEM characterization of CH-NPs revealed the integration of C-NPs with HA-NPs forming CH-NPs nanostructure containing both inorganic and organic materials simulating that of the native bone matrix. This finding is congruent with that of Prajatelistia and colleagues (2015), who demonstrated the formation of CH-NPs using TEM analysis. Our study showed negatively charged $\mathrm{CH}-\mathrm{NPs}$ as indicated from zeta potential analysis. This finding comes in contrast to the study of Uskoković and Desai (2014), who reported the formation of positively charged CH-NPs with zeta potential of $3.9 \mathrm{mV}$. This subtle difference in characteristics between our nanostructure and that in the previous study could be due to the variation in surface chemistry and particle size. It is assumed that nanoparticles obtained from different manufacturers have slight variations in surface chemistry and degree of crystallinity that may affect their zeta potential values.

Microscopic follow up and flow cytometry characterization of the rat BM-MSCs confirmed the identity of MSCs. These findings are in correspondence with those of Muniswami et al. (2018) and Yusop et al. (2018). Furthermore, the isolated BM-MSCs were further identified by their tri-lineage differentiation capacity (adipogenic, chondrogenic and osteogenic). BM-MSCs were successfully differentiated into adipocytes and this comes in line with that of Bayati et al. (2013) and Yusop et al. (2018). Moreover, alizarin red $\mathrm{S}$ staining documented the osteogenic differentiation of BM-MSCs. In addition, alcian blue staining of BM-MSCs emphasized the successful chondrogenic differentiation of BM-MSCs. These results are greatly supported by those of the study of Sangeetha et al. (2017).

According to MTT results, treatment of BM-MSCS with HA-NPs at the studied concentrations, particularly at $10 \mu \mathrm{g} / \mathrm{ml}$, did not cause any significant toxicity to BM-MSCs versus control cells. This finding is greatly supported by that of Remya et al. (2014), who demonstrated that the percentage viability of mouse BM-MSCs treated with $10 \mu \mathrm{g} / \mathrm{ml}$ of rod-shaped HANPs is comparable to that of the negative control.

High concentrations of Au-NPs have been shown to drive the cells towards apoptotic pathways (Connor et al. 2005), and too low concentrations of Au-NPs have been found to be not effective for cell differentiation. Therefore, the optimum concentration of $\mathrm{Au}-$ NPs was chosen according to MTT assay to be evaluated later on BM-MSCs behavior regarding cell viability and differentiation. It was established that $5 \mu \mathrm{g} / \mathrm{ml}$ of Au-NPs did not display any obvious toxicity towards BM-MSCs over $48 \mathrm{~h}$ and $72 \mathrm{~h}$ incubation periods. This observation is in accordance with that of the study of Ko et al. (2015), who mentioned that spherical Au-NPs of $50 \mathrm{~nm}$ size have no significant toxicity on adipose tissue derived-MSCs (ADSCs) over the course of 7 days incubation.

Likewise, BM-MSCs exposed to a concentration of $5 \mu \mathrm{g} / \mathrm{ml}$ of Au/HA-NPs did not show any significant cytotoxicity after $48 \mathrm{~h}$ and $72 \mathrm{~h}$ incubation. This result is in harmony with that of Ferreira dos Santos et al. (2015), who reported that human MSCs subjected to a range of Au/HA-NPs concentrations $(1-100 \mu \mathrm{g} / \mathrm{ml})$ did not display any alteration in cell viability compared with the control cells. 
In a similar pattern, neither $\mathrm{CH}-\mathrm{NPs}$ nor C-NPs exert any significant toxicity to BM-MSCs at $5 \mu \mathrm{g} / \mathrm{ml}$ concentration after $48 \mathrm{~h}$ and $72 \mathrm{~h}$ incubation. This finding is on par with that of Chen et al. (2012), who observed that murine calvarial osteoblasts (MC3T3E1) seeded with chitosan/nano-hydroxyapatite/collagen scaffold did not induce any prominent toxicity when compared to the control cells.

In order to assess the functional features of the differentiated BM-MSCs into osteoblasts, we determined the gene expression levels of Runx-2 and BMP2. Runx-2 is regarded as the earliest transcription factor determinant for directing MSCs towards osteoblast lineage ( $\mathrm{Li}$ et al. 2016). Runx-2 is known to be governed by mitogen-activated protein kinase (MAPK) signaling pathway (Xiao et al. 2000), which in turn activates other osteoblast-specific gene expression such as osteopontin (OPN), osteocalcin (OCN) and collagen type I alpha (Col-1 $\alpha)$ essential for the functional entity of osteoblasts (Komori 2003; Yi et al. 2010). BMP-2 is a member of the transforming growth factor beta (TGF- $\beta$ ) superfamily acting as a cell-cell signaling molecule during bone formation and repair (Benoit et al. 2007). BMP-2 signaling is required for the activation of Runx-2 through the Smad and MAPK intracellular signaling modules (Phimphilai et al. 2006; Khatiwala et al. 2009). BMP activates the downstream Smad-dependent signaling pathway. Activated BMP receptors phosphorylate Smad1/5/8, which then combine with Smad 4 forming a complex transported to the nucleus, where it regulates the transcriptional activation of downstream genes, including Runx-2 and osterix. BMP-2 also activates a series of Smad-independent signaling pathways, including MAPK pathway components such as p38, c-Jun N-terminal kinase (JNK), and extracellular signal-regulated kinase (ERK). These pathways are implicated in stimulating BMP-mediated osteogenic process (Zhang et al. 2014b). Moreover, BMPs are accountable for enhancing osteoblastic differentiation and subsequent bone matrix mineralization through promotion of the expression of bone structural proteins, such as Col-1 $\alpha$ and OCN (Yi et al. 2010).

The current results indicated that incubating BMMSCs in OS medium alone led to significant upregulation of Runx-2 and insignificant over-expression of BMP-2 gene expression levels as compared to undifferentiated BM-MSCs. This finding is greatly supported by the study of Wang and co-workers (2017a), who reported that the Runx-2 gene expression level is significantly up-regulated in BM-MSCs cultured in the osteogenic medium (dexamethasone, ascorbic acid and $\beta$-glycerophosphate) as compared to that cultured in basic medium without osteoinductive supplements. Such bioactive supplements in the osteogenic medium act synergistically to stimulate the osteogenic differentiation through regulating several regulatory mechanisms. Dexamethasone, a synthetic glucocorticoid, acts to amplify the expression level of Runx-2 via mediating the transcriptional activation of $\mathrm{WNT} / \beta$-catenin signaling axis. Hamidouche and his colleagues (2008) observed that four and a half LIM domains protein 2 (FHL-2) gene expressions is stimulated in response to dexamethasone treatment as a result of interaction with a glucocorticoid response element in the promoter of FHL-2. After that, FHL-2 binds to $\beta$-catenin leading to $\beta$-catenin translocation to the nucleus, where it binds to $\mathrm{T}$ cell factor/lymphoid enhancer factor-1 (TCF/ LEF-1), resulting in transcriptional activation of Runx-2. Ascorbic acid induces the osteogenic differentiation via increasing the secretion of Col-1 $\alpha$ into the extracellular matrix leading to $\alpha 2 \beta 1$ integrins binding to Col- $1 \alpha$, which in turn resulting in extracellular-related kinase (ERK1/2) phosphorylation in the MAPK signaling cascade, followed by translocation of the phosphorylated ERK1/2 into the nucleus, where it binds to Runx-2, resulting in its activation and subsequent stimulation of other osteogenic gene expression (Wang et al. 2017a). Since ascorbic acid acts as a cofactor for enzymes responsible for proline and lysine hydroxylation in pro-collagen, resulting in the formation of collagen helical chain (Vater et al. 2011). $\beta$-glycerophosphate was found to facilitate matrix mineralization process via donating phosphate group needed to produce the hydroxyapatite mineral $\left(\mathrm{Ca}_{10}(\mathrm{PO} 4)_{6}(\mathrm{OH})_{2}\right)$ and promote osteoblast-related gene expression via phosphorylation of ERK. Phosphate acts as an intracellular signaling molecule entering the cell, resulting in ERK signaling pathway activation, which in turn leads to stimulation of other osteogenic gene expression such as BMP-2 (Wang et al. 2017a). Moreover, Tada et al. (2011) commented that phosphate stimulates the transcriptional activation of BMP-2 via inducing the cyclic-AMP/protein kinase-A pathway.

The current data showed that incubation of BMMSCs in OS medium containing HA-NPs results in 
insignificant up-regulation in the expression levels of Runx-2 and BMP-2 genes as compared to that incubated in OS medium alone. These data are in conformity with the study of Huang et al. (2012), who documented that rat BM-MSCs treated with OS medium containing $200 \mu \mathrm{g} / \mathrm{ml}$ nano-hydroxyapatite (n-HA) exhibit significant up-regulation of Runx-2 gene expression level. This could be going back to the virtue that $\mathrm{n}$-HA modifies the microenvironments of the cell culture which mostly affect MSCs differentiation (Yuan et al. 2011). Since n-HA acts through adsorbing proteins forming a neo-matrix and hence stimulating osteogenesis. Moreover, the results of the study of Kim et al. (2011) demonstrated that seeding rat BM-MSCs with poly (propylene fumarate) (PPF)/ hydroxyapatite nanocomposite scaffolds greatly motivated their osteogenic differentiation through inducing the over-expression of BMP-2 and Runx-2. This might be explained by the possibility that BMP-2 activation leads to Runx-2 stimulation via both the Smad and MAPK pathways (Lian et al. 2006; Zhang et al. 2014b).

Gene profiling results indicated that the expression of Runx-2 and BMP-2 are significantly up-regulated upon the incubation of BM-MSCs with Au-NPs in combination with OS medium versus that incubated in OS medium alone. This finding is in harmony with the study of Zhang et al. (2014a), who mentioned that 20-nm and 40-nm Au-NPs stimulated the differentiation and matrix mineralization of mouse primary osteoblasts through the up-regulation of both Runx-2 and BMP-2 gene expression levels. Such up-regulation is mediated via the activation of extracellular signal-regulated kinase (ERK)/MAPK cascade.

Moreover, Yi and co-workers (2010) showed three folds increase in Runx-2 and BMP-2 gene expression when MSCs are treated with Au-NPs, indicating that $\mathrm{Au}-\mathrm{NPs}$ preferentially promote the differentiation of MSCs towards the osteoblastic phenotype. They supposed that Au-NPs interact with the cell membrane, enter the cell by receptor-mediated endocytosis into the cytoplasm where they bind with cytoplasmic proteins leading to the induction of osteogenic differentiation via activating the $\mathrm{p} 38$ MAPK pathway. The activation of p38 MAPK signaling pathway leads to the up-regulation of Runx-2 (Wang et al. 2002) with consequent up-regulation of Col-1 $\alpha$ and BMP-2 at early stages of differentiation (Liu et al. 2010b), hence, motivating MSCs differentiation towards osteogenic lineage. Incubating cells in media containing nanomaterials leads to the adsorption of serum proteins to their surface, triggering their entry into cells via endocytosis (Khan et al. 2007; Verma and Stellacci 2010). These proteins, adsorbed on Au-NPs surface, interfere with the ERK signaling axis, which is known to be essential for osteogenic differentiation of MSCs (Zhang et al. 2014a).

The results of the BM-MSCs incubated with $\mathrm{Au} /$ HA-NPs plus OS medium denoted a significant upregulation of both Runx-2 and BMP-2 gene expression levels when compared with that cultured in OS medium alone. These findings echo those of Xia and his colleagues (2018), who reported that gold nanoparticles incorporated with Calcium Phosphate cement drive the cells towards osteogenic lineage via upregulation of the gene expression level of Runx-2.

Chitosan-based scaffolds have been previously reported to support the attachment and proliferation of both osteoblasts and osteoclasts, which represent an emerging approach for bone tissue engineering applications (Jones et al. 2009). However, such scaffolds alone are not sufficient to mimic the natural bone architecture. As they only work to induce the organic phase and hence represent an incomplete matrix. Therefore, the incorporation of hydroxyapatite nanocrystalline, as an inorganic phase, within these scaffolds may represent a perfect candidate comparable to the natural bone microenvironment in order to support bone regeneration.

Our results demonstrated that incubating BMMSCs with C-NPs-containing OS medium resulted in insignificant alteration in the expression of Runx-2 and BMP-2 gene expression level relative to that incubated in OS medium alone. This finding is in compliance with that of Dhivya et al. (2015), who reported that nano-hydroxyapatite-free chitosan composite insignificantly stimulates the expression of Runx-2 gene in osteoblast-like cells as compared to that containing nanohydroxyapatite. Ma et al. (2018) demonstrated that culturing rat osteoblasts on titanium implants coated with nano-HA/chitosan composite stimulates osteogenesis through activation of the focal adhesion kinase (FAK), which in turn stimulates the activation of BMP-2/Smad pathway. This could be attributed to the favorable wettability of chitosan providing a support for osteoblasts adhesion and osteoblasts differentiation via regulating wettabilitysensitive integrins expression. This leads to increased 
expression of $\alpha 1, \alpha 2$ and $\beta 1$ integrin subunits, which in turn affects integrin-BMP/Smad signaling module through phosphorylation of FAK, hence, activating the transcription factors Smad1/5/8 via BMP-2 signaling activation, leading to the transcriptional activation of Runx-2, resulting in stimulation of osteoblastic differentiation.

In the current study, we incorporate chitosan with HA-NPs in the presence of OS medium to improve the material's osteoinductive potential and support the adhesion of MSCs over time in culture. Incorporation of HA-NPs with chistosan nanoparticles triggered significant over-expression of BMP-2 in concomitant with insignificant up-regulation of Runx-2 gene expression levels as compared to BM-MSCs incubated in OS medium alone. This finding is in coherence with that of Peng et al. (2012), who recorded that nHAchitosan scaffolds support better attachment and proliferation of MSCs and up-regulate osteogenic gene markers, including Col- $1 \alpha$, Runx-2, ALP, and OCN in the absence of OS medium. A possible mechanism responsible for such effect on MSCs proliferation and osteogenic differentiation may be due to the nanoscale surface structure of these scaffolds, which enhances adhesion of cells to HANPs. Such scale also permits much great surface area and reactivity, which in turn induces the scaffold's ability to guide cell mitogenic behavior ( $\mathrm{Hu}$ et al. 2014).

The osteoinductive effect of CH-NPs on MSCs may be ascribed to the sustained dissolution and reprecipitation of Calcium and Phosphate ions once Calcium Phosphate-based scaffolds get hydrated into cell culture media. This generates intense carbonated apatite layer over the scaffold surface similar to that of the native bone and hence may stimulate functional osteogenic differentiation and matrix mineralization (Chen et al. 2012).

Alizarin red $\mathrm{S}$ assay has been extensively employed to identify the calcium-rich deposits formed during MSCs differentiation as a tool for evaluating their osteogenic differentiation capacity (Macri-Pellizzeri et al. 2018). The current data showed that Calcium Phosphate precipitates are detected by alizarin red $\mathrm{S}$ stain in the BM-MSCs cultured in OS medium coupled with HA-NPs. This is in concert with the report by Lock and Liu (2011), which proved enhanced calcium deposition by human BM-MSCs upon incubation with nano-hydroxyapatite.
In the present study, alizarin red $\mathrm{S}$ staining revealed the presence of Calcium Phosphate precipitates in the cellular matrix of BM-MSCs cultured in OS medium containing Au-NPs. This result converges with the study conducted by Li et al. (2016), who demonstrated that the incubation of human MSCs with Au-NPs leads to the enhancement of the osteogenic differentiation documented by remarkable Calcium Phosphate deposition upon staining with alizarin red $\mathrm{S}$. This study suggested that the mechanism underlying the promotion of the osteogenic differentiation of hMSCs by AuNPs may be due to the regulation of Yes-associated protein (YAP) activity.

In our study, BM-MSCs exposed to Au/HA-NPs coupled with OS medium displayed an increased matrix mineralization confirmed by alizarin red staining of calcium precipitates. This corroborates the finding of Xia et al. (2018), who demonstrated that incorporation of gold nanoparticles with Calcium Phosphate greatly induces more calcium deposition. Choi et al. (2015) suggested that chitosan-conjugated $\mathrm{Au}-\mathrm{NPs}$ promotes the ostegenic differentiation of ADSCs via stimulating $\mathrm{Wnt} / \beta$-catenin signaling cascade known to be implicated in osteogenic differentiation of MSCs (Guidotti et al. 2013), leading to the increase in calcium deposition.

Our results detected the formation of mineralized calcium nodules after incubating BM-MSCs with $\mathrm{CH}$ NPs containing OS medium. This finding coincides with that of Rogina and co-workers (2017), who emphasized the great impact of chitosan-hydroxyapatite scaffolds on human MSCs osteogenesis as evidenced by the strong calcium deposits staining. On the contrary, culturing of BM-MSCs in OS medium supplemented with C-NPs shows slight calcium deposition as compared to that of HAincorporated chitosan composite. This finding is in harmony with that of Chen et al. (2012), who observed that mouse calvarial preosteoblasts incubated with chitosan-only scaffold show slight osteoblasts mineralization, as shown by alizarin assay, compared to those cultured with nano-hydroxyapatite-conjugated chitosan scaffold,since chitosan scaffold insignificantly stimulates the osteogenic markers production, including ALP, Col-1 $1 \alpha$, OCN and Runx-2. 


\section{Conclusion}

The present study emphasizes the potency of the studied nanomaterials in triggering BM-MSCs towards osteogenic lineage. Noteworthy, the dominant osteoinductive effect was observed for Au-NPs followed by Au/HA-NPs and CH-NPs, as evidenced by gene expression analysis and alizarin red assay, which afforded a strong document for their promising role to be used for bone tissue engineering.

Acknowledgements The authors gratefully acknowledge the financial support of the National Research Centre, Egypt.

Funding This work was financially supported by the National Research Centre, Egypt (Thesis fund No. 71511).

\section{Compliance with ethical standards}

Conflict of interest The authors declare that they have no conflict of interest.

\section{References}

Akahane M, Ueha T, Shimizu T, Inagaki Y, Kido A, Imamura T, Kawate K, Tanaka Y (2012) Increased osteogenesis with hydroxyapatite constructs combined with serially-passaged bone marrow-derived mesenchymal stem cells. Stem Cell Discov 2:133-140

Bayati V, Hashemitabar M, Gazor R, Nejatbakhsh R, Bijannejad D (2013) Expression of surface markers and myogenic potential of rat bone marrow and adipose-derived stem cells: a comparative study. Anat Cell Biol 46:113-121

Benoit DSW, Collins SD, Anseth KS (2007) Multifunctional hydrogels that promote osteogenic human mesenchymal stem cell differentiation through stimulation and sequestering of bone morphogenic protein 2. Adv Funct Mater 17:2085-2093

Cai Y, Liu Y, Yan W, Hu Q, Tao J, Zhang M, Shic Z, Tang R (2007) Role of hydroxyapatite nanoparticle size in bone cell proliferation. J Mater Chem 17:3780-3787

Chen Y, Huang Z, Li X, Li S, Zhou Z, Zhang Y, Feng Q, Yu B (2012) In vitro biocompatibility and osteoblast differentiation of an injectable chitosan/nano-hydroxyapatite/collagen scaffold. J Nanomater 2012:1-6. https://doi.org/10. $1155 / 2012 / 401084$

Chen H, Dorrigan A, Saad S, Hare DJ, Cortie MB, Valenzuela SM (2013) In vivo study of spherical gold nanoparticles: inflammatory effects and distribution in mice. PLoS ONE 8:e58208

Choi SY, Song MS, Ryu PD, Lam ATN, Joo S-W, Lee SY (2015) Gold nanoparticles promote osteogenic differentiation in human adipose-derived mesenchymal stem cells through the Wnt/ $\beta$-catenin signaling pathway. Int $\mathrm{J}$ Nanomed 10:4383-4392
Connor EE, Mwamuka J, Gole A, Murphy CJ, Wyatt MD (2005) Gold nanoparticles are taken up by human cells but do not cause acute cytotoxicity. Small 1:325-327

Crisan L, Crisan B, Soritau O, Baciut M, Biris AR, Baciut G, Lucaciu O (2015) In vitro study of biocompatibility of a graphene composite with gold nanoparticles and hydroxyapatite on human osteoblasts. J Appl Toxicol 35:1200-1210

Dhivya S, Saravanan S, Sastry TP, Selvamurugan N (2015) Nanohydroxyapatite-reinforced chitosan composite hydrogel for bone tissue repair in vitro and in vivo. J Nanobiotechnol 13:40

Eliaz N, Metoki N (2017) Calcium phosphate bioceramics: a review of their history, structure, properties, coating technologies and biomedical applications. Mater (Basel) 10:e334. https://doi.org/10.3390/ma10040334

Ferreira dos Santos C, Gomes PS, Almeida MM, Willinger M, Franke R, Fernandescd MH, El Costa M (2015) Golddotted hydroxyapatite nanoparticles as multifunctional platforms for medical applications. RSC Adv 5:69184-69195

Fitzsimmons REB, Mazurek MS, Soos A, Simmons CA (2018) Mesenchymal stromal/stem cells in regenerative medicine and tissue engineering. Stem Cells Int 2018:1-16. https:// doi.org/10.1155/2018/8031718

Freese C, Gibson MI, Klok HA, Unger RE, Kirkpatrick CJ (2012a) Size- and coating-dependent uptake of polymercoated gold nanoparticles in primary human dermal microvascular endothelial cells. Biomacromol 13:1533-1543

Freese C, Uboldi C, Gibson MI, Unger RE, Weksler BB, Romero IA, Couraud P-O, Kirkpatrick CJ (2012b) Uptake and cytotoxicity of citrate-coated gold nanospheres: comparative studies on human endothelial and epithelial cells. Part. Fibre Toxicol 9:23

Grenha A, Seijo B, Remunan-Lopez C (2005) Microencapsulated chitosan nanoparticles for lung protein delivery. Eur J Pharm Sci 25:427-437

Guidotti S, Facchini A, Platano D, Olivotto E, Minguzzi M, Trisolino G, Filrdo G, Cetrullo S, Tantini B, Martucci E, Facchini A, Flamigni F, Borzi RM (2013) Enhanced osteoblastogenesis of adipose-derived stem cells on spermine delivery via beta-catenin activation. Stem Cells Dev 22:1588-1601

Hamidouche Z, Hay E, Vaudin P, Charbord P, Schule R, Marie PJ, Fromigue O (2008) FHL2 mediates dexamethasoneinduced mesenchymal cell differentiation into osteoblasts by activating Wnt/beta-catenin signalingdependent Runx2 expression. Faseb J 22:3813-3822

Heo DN, Ko WK, Bae MS, Lee JB, Lee D-W, Byun W, Lee CH, Kim E-C, Jung B-Y, Kwon K (2014) Enhanced bone regeneration with a gold nanoparticle-hydrogel complex. J Mater Chem B Mater Biol Med 2:1584-1594

Hu J, Zhou Y, Huang L, Liu J, Lu H (2014) Effect of nanohydroxyapatite coating on the osteoinductivity of porous biphasic calcium phosphate ceramics. BMC Musculoskelet Disord 15:114

Huang GT, Gronthos S, Shi S (2009) Mesenchymal stem cells derived from dental tissues vs. those from other sources: their biology and role in regenerative medicine. J Dent Res 88:792-806 
Huang Y, Zhou G, Zheng L, Liu H, Niu X, Fan Y (2012) Micro-/nano- sized hydroxyapatite directs differentiation of rat bone marrow derived mesenchymal stem cells towards an osteoblast lineage. Nanoscale 4:2484-2490

Hunter RJ (1981) Zeta potential in colloid science: principles and applications. Academic Press, New York

Iswanti FC, Nurulita I, Djauzi S, Sadikin M, Witarto AB, Yamazaki T (2019) Preparation, characterization, and evaluation of chitosan-based nanoparticles as CpG ODN carriers. Biotechnol Biotechnol Equip. https://doi.org/10. 1080/13102818.2019.1578690

Jones GL, Motta A, Marshall MJ, El Haj AJ, Cartmell SH (2009) Osteoblast: osteoclast co-cultures on silk fibroin, chitosan and PLLA films. Biomaterials 30:5376-5384

Khan JA, Pillai B, Das TK, Singh Y, Maiti S (2007) Molecular effects of uptake of gold nanoparticles in HeLa cells. ChemBioChem 8:1237-1240

Khatiwala CB, Kim PD, Peyton SR, Putnam AJ (2009) ECM compliance regulates osteogenesis by influencing MAPK signaling downstream of RhoA and ROCK. J Bone Miner Res 24:886-898

Kim K, Dean D, Lu A, Mikos AG, Fisher JP (2011) Early osteogenic signal expression of rat bone marrow stromal cells is influenced by both hydroxyapatite nanoparticle content and initial cell seeding density in biodegradable nanocomposite scaffolds. Acta Biomater 7:1249-1264. https://doi.org/10.1016/j.actbio.2010.11.007

Ko WK, Heo DN, Moon HJ, Lee SJ, Bae MS, Lee JB, Sun IC, Jeon HB, Park HK, Kwon IK (2015) The effect of gold nanoparticle size on osteogenic differentiation of adiposederived stem cells. J Colloid Interface Sci 438:68-76. https://doi.org/10.1016/j.jcis.2014.08.058

Komori T (2003) Requisite roles of Runx 2 and Cbfb in skeletal development. J Bone Miner Metab 21:193-197

Levengood SKL, Zhang M (2014) Chitosan-based scaffolds for bone tissue engineering. J Mater Chem B 2:3161-3184. https://doi.org/10.1039/C4TB00027G

Li J, Li JJ, Zhang J, Wang X, Kawazoea N, Chen G (2016) Gold nanoparticle size and shape influence on osteogenesis of mesenchymal stem cells. Nanoscale 8:7992-8007

Lian JB, Stein GS, Javed A, Van Wijnen AJ, Stein L, Montecino M, Hassan MQ, Gaur T, Lengner CJ, Young DW (2006) Networks and hubs for the transcriptional control of osteoblastogenesis. Rev Endocr Metab Disord 7:1-16

Linard C, Brachet M, L'homme B, Strup-Perrot C, Busson E, Bonneau M, Lataillade JJ, Bey E, Benderitter M (2018) Long-term effectiveness of local BM-MSCs for skeletal muscle regeneration: a proof of concept obtained on a pig model of severe radiation burn. Stem Cell Res Ther 9:299. https://doi.org/10.1186/s13287-018-1051-6

Liu DD, Yi CQ, Zhang DW, Zhang JC, Yang MS (2010a) Inhibition of proliferation and differentiation of mesenchymal stern cells by carboxylated carbon nanotubes. ACS Nano 4:2185-2195

Liu DD, Zhang JC, Yi CQ, Yang M (2010b) The effects of gold nanoparticles on the proliferation, differentiation, and mineralization function of MC3T3-E1 cells in vitro. Chin Sci Bull 55:1013-1019. https://doi.org/10.1007/s11434010-0046-1
Livak KJ, Schmittgen TD (2001) Analysis of relative gene expression data using real-time quantitative PCR and the $2^{(- \text {Delta Delta C(T)) }}$ method. Methods 25:402-408

Lock J, Liu H (2011) Nanomaterials enhance osteogenic differentiation of human mesenchymal stem cells similar to a short peptide of BMP-7. Int J Nanomed 6:2769-2777

Lu Z, Roohani-Esfahani S-I, Kwok PCL, Zreiqat H (2011) Osteoblasts on rod shaped hydroxyapatite nanoparticles incorporated pcl film provide an optimal osteogenic niche for stem cell differentiation. Tissue Eng Part A 17:1651-1661

Ma X-Y, Feng Y-F, Wang T-S, Le WI, Li X, Zhou D-P, Wen X-X, Yu H-L, Xiang L-B, Wang L (2018) Involvement of FAK-mediated BMP-2/Smad pathway in mediating osteoblast adhesion and differentiation on nano-HA/chitosan composite coated titanium implant under diabetic conditions. Biomater Sci 6:225-238

Macri-Pellizzeri L, De Melo N, Ahmed I, Grant D, Scammell B, Sottile V (2018) Live quantitative monitoring of mineral depositionin stem cells using tetracycline hydrochloride. Tissue Eng Part C Methods 24:171-178

Mahla RS (2016) Stem cells applications in regenerative medicine and disease therapeutics. Int $\mathrm{J}$ Cell Biol 2016:6940283

Mansour SF, El-dek SI, Ahmed MK (2017) Physico-mechanical and morphological features of zirconia substituted hydroxyapatite nanocrystals. Sci Rep 7:43202

Mauricio MD, Guerra-Ojeda S, Marchio P, Valles SL, Aldasoro M, Escribano-Lopez I, Herance JR, Rocha M, Vila JM, Victor VM (2018) Nanoparticles in medicine: a focus on vascular oxidative stress. Oxid Med Cell Longev 2018:6231482. https://doi.org/10.1155/2018/6231482

Müller P, Lemcke H, David R (2018) Stem cell therapy in heart diseases - cell types, mechanisms and improvement strategies. Cell Physiol Biochem 48:2607-2655. https:// doi.org/10.1159/000492704

Muller KH, Motskin M, Philpott AJ, Routh AF, Shanahan CM, Duer MJ, Skepper JN (2014) The effect of particle agglomeration on the formation of a surface-connected compartment induced by hydroxyapatite nanoparticles in human monocyte-derived macrophages. Biomater 35:1074-1088.

Muniswami DM, Kanthakumar P, Kanakasabapathy I, Tharion G (2018) Motor recovery after transplantation of bone marrow mesenchymal stem cells in rat models of spinal cord injury. Ann Neurosci 25:126-140

Naruphontjirakul P, Tsigkou O, Li S, Porter AE, Jones JR (2019) Human mesenchymal stem cells differentiate into an osteogenic lineage in presence of strontium containing bioactive glass nanoparticles. Acta Biomater 90:373-392. https://doi.org/10.1016/j.actbio.2019.03.038

Nguyen AK, Patel R, Noble JM, Zheng J, Narayan RJ, Kumar G, Goering PL (2019) Effects of subcytotoxic exposure of silver nanoparticles on osteogenic differentiation of human bone marrow stem cells. Appl In Vitro Toxicol 5:123-133. https://doi.org/10.1089/aivt.2019.0001

Niu W, Guo Y, Xue Y, Chen M, Wang M, Cheng W, Lei B (2019) Monodisperse branched molybdenum-based bioactive nanoparticles significantly promote osteogenic differentiation of adipose-derived stem cells. Part Part Syst 
Charact 36:1900105. https://doi.org/10.1002/ppsc. 201900105

Okada M, Matsumoto T (2015) Synthesis and modification of apatite nanoparticles for use in dental and medical applications. Jpn Dent Sci Rev 51:85-95

Peng H, Yin Z, Liu H, Chen X, Feng B, Yuan H, Su B, Ouyang H, Zhang Y (2012) Electrospun biomimetic scaffold of hydroxyapatite/chitosan supports enhanced osteogenic differentiation of mMSCs. Nanotechnology 23:485102. https://doi.org/10.1088/0957-4484/23/48/485102

Phimphilai M, Zhao Z, Boules H, Roca H, Franceschi RT (2006) BMP signaling is required for RUNX 2-dependent induction of the osteoblast phenotype. J Bone Miner Res 21:637-646

Pissuwan D, Cortie CH, Valenzuela SM, Cortie MB (2007a) Gold nanosphere antibody conjugates for hyperthermal therapeutic applications. Gold Bull 40:121-129

Pissuwan D, Valenzuela SM, Killingsworth MC, Xu XD, Cortie MB (2007b) Targeted destruction of murine macrophage cells with bioconjugated gold nanorods. J Nanopart Res 9:1109-1124

Prajatelistia E, Lim C, Oh DX, Jun SH, Hwang DS (2015) Chitosan and hydroxyapatite composite cross-linked by dopamine has improved anisotropic hydroxyapatite growth and wet mechanical properties. Eng Life Sci 15:254-261

Remya NS, Syama S, Gayathri V, Varma HK, Mohanan PV (2014) An in vitro study on the interaction of hydroxyapatite nanoparticles and bone marrow mesenchymal stem cells for assessing the toxicological behavior. Colloids Surf B Biointerfaces 117:389-397

Rodríguez-Vázquez M, Vega-Ruiz B, Ramos-Zúñiga R, Saldaña-Koppel DA, Quiñones-Olvera LF (2015) Chitosan and its potential use as a scaffold for tissue engineering in regenerative medicine. Biomed Res Int 2015:821279

Rogina A, Antunović M, Pribolšan L, Mihalić KC, Vukasović A, Ivković A, Marijanović I, Ferrer GG, Ivanković M, Ivanković H (2017) human mesenchymal stem cells differentiation regulated by hydroxyapatite content within chitosan-based scaffolds under perfusion conditions. Polymers 9:387

Roohani-Esfahani SI, Nouri-Khorasani S, Lu Z, Appleyard R, Zreiqat H (2010) The influence hydroxyapatite nanoparticle shape and size on the properties of biphasic calcium phosphate scaffolds coated with hydroxyapatite PCL composites. Biomaterials 31:5509

Sangeetha P, Maiti SK, Mohan D, Shivaraju S, Raguvaran R, Abu Rafee M, Bindhuja BV, Kumar N, Raguvanshi PDS (2017) Mesenchymal stem cells derived from rat bone marrow (rBM-MSCs): techniques for isolation, expansion and differentiation. J Stem Cell Res Ther 3:272-277. https://doi.org/10.15406/jsrt.2017.03.00101

Singh P, Pandit S, Mokkapati VRSS, Garg A, Ravikumar V, Mijakovic I (2018) Gold nanoparticles in diagnostics and therapeutics for human cancer. Int J Mol Sci 19:1979. https://doi.org/10.3390/ijms19071979

Sobczak-Kupiec A, Tyliszczak B, Krupa-Żuczek K, Malina D, Piątkowski M, Wzorek Z (2014) Gold nanoparticles as a modifying agent of ceramic-polymer composites. Arch Metall Mater 59:1009

Tada H, Nemoto E, Foster BL, Somerman MJ, Shimauchi H (2011) Phosphate increases bone morphogenetic protein-2 expression through cAMP dependent protein kinase and ERK1/2 pathways in human dental pulp cells. Bone 48:1409-1416

Ullah I, Subbarao RB, Rho GJ (2015) Human mesenchymal stem cells - current trends and future prospective. Biosci Rep 35:e00191. https://doi.org/10.1042/BSR20150025

Uskoković V, Desai TA (2014) In vitro analysis of nanoparticulate hydroxyapatite/chitosan composites as potential drug delivery platforms for the sustained release of antibiotics in the treatment of osteomyelitis. J Pharm Sci 103:567-579. https://doi.org/10.1002/jps.23824

Van Meerloo J, Kaspers GJL, Cloos J (2011) Cell sensitivity assays: the MTT assay. Methods Mol Biol 731:237-245. https://doi.org/10.1007/978-1-61779-80-5_20

Vandiver J, Dean D, Patel N, Bonfield W, Ortiz C (2005) Nanoscale variation in surface charge of synthetic hydroxyapatite detected by chemically and spatially specific high-resolution force spectroscopy. Biomaterials 26:271-283

Vater C, Kasten P, Stiehler M (2011) Culture media for the differentiation of mesenchymal stromal cells. Acta Biomater 7:463-477

Verma A, Stellacci F (2010) Effect of surface properties on nanoparticle-cell interactions. Small 6:12-21

Wan A-J, Sun Y, Li W-T, Li H-L (2007) transmission electron microscopy and electron diffraction study of BSA-loaded quaternized chitosan nanoparticles. J Biomed Mater Res B Appl Biomater 86:197-207

Wang FS, Wang CJ, Sheen-Chen SM, Kuo YR, Chen RF, Yang KD (2002) Superoxide mediates shock wave induction of ERK-dependent osteogenic transcription factor (CBFA1) and mesenchymal cell differentiation toward osteoprogenitors. J Biol Chem 277:10931-10937

Wang C, Meng H, Wang X, Zhao C, Peng J, Wang Y (2016) Differentiation of bone marrow mesenchymal stem cells in osteoblasts and adipocytes and its role in treatment of osteoporosis. Med Sci Monit 22:226-233

Wang C, Cao X, Zhang Y (2017a) A novel bioactive osteogenesis scaffold delivers ascorbic acid, $\beta$-glycerophosphate, and dexamethasone in vivo to promote bone regeneration. Oncotarget 8:31612-31625. https://doi.org/10.18632/ oncotarget.15779

Wang Q, Chen B, Ma F, Lin S, Cao M, Li Y, Gu N (2017b) Magnetic iron oxide nanoparticles accelerate osteogenic differentiation of mesenchymal stem cells via modulation of long noncoding RNA INZEB2. Nano Res 10:626-642. https://doi.org/10.1007/s12274-016-1322-4

Woodbury D, Schwarz EJ, Prockop DJ, Black IB (2000) Adult rat and human bone marrow stromal cells differentiate into neurons. J Neurosci Res 61:364-370

Xia Y, Chen H, Zhang F, Bao C, Weir MD, Reynolds MA, Ma J, $\mathrm{Gu} \mathrm{N}, \mathrm{Xu}$ HHK (2018) Gold nanoparticles in injectable calcium phosphate cement enhance osteogenic differentiation of human dental pulp stem cells. Nanomedicine 14:35-45

Xiao G, Jiang D, Thomas P, Benson MD, Guan K, Karsenty G, Franceschi RT (2000) MAPK pathways activate and phosphorylate the osteoblast-specific transcription factor, Cbfa1. J Biol Chem 275:4453-4459

Yang K, Cao W, Hao X, Xue X, Zhao J, Liu J, Zhao Y, Meng J, Sun B, Zhang J (2013) Metallofullerene nanoparticles 
promote osteogenic differentiation of bone marrow stromal cells through BMP signaling pathway. Nanoscale 5:1205-1212

Yao W, Lane NE (2015) Targeted delivery of mesenchymal stem cells to the bone. Bone 70:62-65

Yao W, Guan M, Jia J, Dai W, Lay YA, Amugongo S, Liu R, Olivos D, Saunders M, Lam KS, Nolta J, Olvera D, Ritchie RO, Lane NE (2013) Reversing bone loss by directing mesenchymal stem cells to bone. Stem Cells 31:2003-2014

Yi C, Liu D, Fong CC, Zhang J, Yang M (2010) Gold nanoparticles promote osteogenic differentiation of mesenchymal stem cells through p38 MAPK pathway. ACS Nano 4:6439-6448. https://doi.org/10.1021/nn101373r

Yuan T, Luo H, Tan J, Fan H, Zhang X (2011) The effect of stress and tissue fluid microenvironment on allogeneic chondrocytes in vivo and the immunological properties of engineered cartilage. Biomaterials 32:6017-6024

Yubao L, Klein CPAT, de Wijn J, Wolke J, de Groot K (1993) Morphology and phase structure of nanograde boneapatitelike rodshaped crystals. In: Ducheyne P, Christiansen D (eds) Bioceramics. Butterworth-Heinemann, Philadelphia, pp 173-178

Yusop N, Battersby P, Alraies A, Sloan AJ, Moseley R, Waddington RJ (2018) Isolation and characterisation of mesenchymal stem cells from rat bone marrow and the endosteal niche: a comparative study. Stem Cells Int 2018:1-14

Zainol I, Zakaria FA, Saliman MR, Derman MA (2008) Preparation and characterisation of chitosan/nano hydroxyapatite composites. J Solid State Sci Technol 16:153-159

Zhang D, Liu D, Zhang J, Fong C, Yang M (2014a) Gold nanoparticles stimulate differentiation and mineralization of primary osteoblasts through the ERK/MAPK signaling pathway. Mater Sci Eng C Mater Biol Appl 42:70-77

Zhang X, Guo J, Zhou Y, Wu G (2014b) The roles of bone morphogenetic proteins and their signaling in the osteogenesis of adipose-derived stem cells. Tissue Eng Part B 20:84-92

Zhao F, Zhao Y, Liu Y, Chang X, Chen C, Zhao Y (2011) Cellular uptake, intracellular trafficking, and cytotoxicity of nanomaterials. Small 7:1322-1337

Publisher's Note Springer Nature remains neutral with regard to jurisdictional claims in published maps and institutional affiliations. 